\title{
Naturalness and dimensional transmutation in classically scale-invariant gravity
}

\author{
Martin B. Einhorn ${ }^{a, b}$ and D.R. Timothy Jones ${ }^{a, c}$ \\ ${ }^{a}$ Kavli Institute for Theoretical Physics, University of California, \\ Santa Barbara, CA 93106-4030, U.S.A. \\ ${ }^{b}$ Michigan Center for Theoretical Physics, University of Michigan, \\ Ann Arbor, MI 48109-1040, U.S.A. \\ ${ }^{c}$ Dept. of Mathematical Sciences, University of Liverpool, \\ Liverpool L69 3BX, U.K. \\ E-mail: meinhorn@umich.edu, drtj@liv.ac.uk
}

ABSTRACT: We discuss the nature of quantum field theories involving gravity that are classically scale-invariant. We show that gravitational radiative corrections are crucial in the determination of the nature of the vacuum state in such theories, which are renormalisable, technically natural, and can be asymptotically free in all dimensionless couplings. In the pure gravity case, we discuss the role of the Gauss-Bonnet term, and we find that Dimensional Transmutation (DT) à la Coleman-Weinberg leads to extrema of the effective action corresponding to nonzero values of the curvature, but such that these extrema are local maxima. In even the simplest extension of the theory to include scalar fields, we show that the same phenomenon can lead to extrema that are local minima of the effective action, with both non-zero curvature and non-zero scalar vacuum expectation values, leading to spontaneous generation of the Planck mass. Although we find an asymptotically free (AF) fixed point exists, unfortunately, no running of the couplings connect the region of DT to the basin of attraction of the AF fixed point. We also find there remains a flat direction for one of the conformal modes. We suggest that in more realistic models AF and DT could be compatible, and that the same scalar vacuum expectation values could be responsible both for DT and for spontaneous breaking of a Grand Unified gauge group.

Keywords: Models of Quantum Gravity, Anomalies in Field and String Theories, SpaceTime Symmetries, Renormalization Group

ArXiv EPrint: 1410.8513 


\section{Contents}

1 Introduction 1

2 The action for pure $R^{2}$ "gravity" $\quad 4$

$\begin{array}{lll}3 & \text { Scale symmetry breaking and naturalness } & 7\end{array}$

4 Dimensional transmutation in $R^{2}$ gravity $\quad 8$

5 Matter: the real scalar field $\quad 15$

6 Additional matter 26

$\begin{array}{lll}7 & \text { Constraints on coupling constants } & 27\end{array}$

8 Conclusions $\quad 30$

$\begin{array}{ll}\text { A Gauss-Bonnet relation } & 31\end{array}$

B Background field method $\quad 32$

$\begin{array}{ll}\text { C Global scale invariance } & 34\end{array}$

D Stability of one-loop effective action 34

E One-loop beta-functions $\quad 36$

\section{Introduction}

Classically scale-invariant and conformally invariant models have attracted great interest in quantum field theory (QFT) for a very long time in a variety of contexts, both phenomenological and theoretical. These symmetries are anomalous in QFT in four-dimensions except in rare circumstances, such as $\mathrm{N}=4$ supersymmetric Yang-Mills theory. This symmetry breaking is inherent in the renormalization process, leading to the concept of scaledependent or running coupling constants.

Why, then, should one be interested in classically scale-invariant theories? One reason is that, in the search for the origin of masses, dimensional transmutation (DT) is a mechanism that can "explain" the appearance of a mass scale from an otherwise massless theory and can lead to definite relationships among masses that are not simply the consequence of internal symmetries. A second motivation is also that classically scale-invariant models that include the metric tensor are renormalizable [1], and their coupling constants are asymptotically free $(\mathrm{AF})$ or asymptotically finite, at least for some range of parameters ${ }^{1}[2-5]$.

\footnotetext{
${ }^{1}$ Ref. [5] contains a detailed review of higher-order gravity. (This is essentially a reproduction of the author's 1986 Ph.D. thesis [hep-th/9510140].) Ref. [6] provides a comprehensive overview by some of the pioneers in the field. Unfortunately, this contains numerous typographical errors in the equations associated with $R^{2}$-gravity.
} 
In principle, such models may provide an ultraviolet (UV) completion of Einstein gravity. They offer the prospect of generating the Planck mass $M_{P}$ dynamically, with consistent physics at energies above $M_{P}$. Even if not the final word, they may provide a perturbatively calculable framework within which some of the puzzles associated with quantum gravity may be given definite answers. Needless to say, such models may also be very important for understanding the very early universe, especially if inflation is an ingredient.

A third motivation is that such theories retain a legacy of their classical scale invariance inasmuch as their symmetry-breaking is "soft" [7-9, 11], i.e., the masses do not suffer from naturalness issues associated with power-law divergences [12].

In the simplest case of massless, scalar $\lambda \phi^{4}$ theory in flat spacetime, the running of the coupling $\lambda(\mu)$ insures that, as $\mu \rightarrow 0$, in fact $\lambda \rightarrow 0$, and the model approaches a free field theory. It also suggests that as $\mu \rightarrow \infty, \lambda(\mu)$ becomes large, so that, above some sufficiently high energy $\Lambda$, the theory becomes strongly coupled. This is frequently interpreted as a sign that, at scales above $\Lambda$ (often associated with the term "Landau pole"), the theory is not simply strongly coupled but incomplete or inconsistent. In other cases, such as Yang-Mills (Y-M) theory or massless QCD, the theory becomes AF at high momentum scales $\mu$, with gauge coupling $g(\mu) \rightarrow 0$ as $\mu \rightarrow \infty$, but strongly coupled below some low-energy scale $\Lambda$. Although not yet rigorously proven, it is firmly believed that the result is gluon condensation or quark confinement, i.e., unlike perturbation theory in which the quanta are massless, the true spectrum of the theory has massive particles whose mass scale is determined by where the effective interaction strength, characterized by $\alpha(\mu)=g^{2}(\mu) / 4 \pi$, becomes sufficiently large (typically, $\alpha(\mu) \sim 1$.) We shall refer to this generically as dimensional transmutation (DT) due to strong interactions.

Finally, there is a third possibility, first discussed by Coleman and Weinberg (CW) [13], in which a classically scale-invariant theory generates a mass scale $\Lambda$ at which a specific relationship among multiple couplings obtains. In the case of scalar electrodynamics, this occurs for $\lambda(\Lambda) \sim \alpha(\Lambda)^{2}$, which can be at weak coupling where perturbation theory may still be a good approximation. This has been called "dimensional transmutation," whereby a massless theory with two or more couplings can be described in terms of a mass scale $\Lambda$ and a single coupling $\alpha(\mu)$ together with a relation that determines the second coupling $\lambda(\Lambda)$ at the specific scale $\Lambda$. If necessary, to distinguish this case from the strong-coupling mechanism characteristic of theories like QCD, we shall refer to this as perturbative or weak-coupling DT.

Among classically scale-invariant theories is higher-order gravity, often referred to as $R^{2}$-gravity, described by a "higher-order" action such as $^{2}$

$$
S_{\mathrm{ho}}=\int d^{4} x \sqrt{g}\left[\frac{1}{2 \alpha} C_{\kappa \lambda \mu \nu}^{2}+\frac{1}{3 \beta} R^{2}+\frac{2}{\gamma} R_{\mu \nu}^{2}\right],
$$

where $C_{\kappa \lambda \mu \nu}$ is the Weyl tensor, $R_{\mu \nu}$ is the Ricci tensor, and $R$ the Ricci scalar. These are the maximum number of scalars of dimension four that can be formed from the Riemann-

\footnotetext{
${ }^{2}$ It is convenient, although probably not necessary, to work with the Euclidean form of the QFT, and we shall do so throughout this paper. For Einstein gravity, the Euclidean Path Integral is not wellunderstood [14], and some of the same issues would apply to $R^{2}$ theories [15]. If the spacetime manifold has boundaries, one needs to supplement this action integral $[16,17]$, but these will not be relevant to our applications.
} 
curvature tensor $R_{\kappa \lambda \mu \nu}$. The three coupling constants $\alpha, \beta, \gamma$ are dimensionless. As mentioned earlier, with a propagator behaving as $1 / q^{4}$, this theory has been shown to be renormalizable and asymptotically free, with or without the addition of a linear term in $R$, a cosmological constant, or, with some weak limitations, with the inclusion of matter.

The low-energy behaviour of this theory is not well understood. If a linear (EinsteinHilbert) term $M^{2} R$ is explicitly added, the perturbative spectrum in flat background has a massive scalar, a massless graviton, and a massive, spin-two ghost [18]. For this reason, the theory is often thought to violate unitarity; it seems as if this model is just a clever way of embedding a Pauli-Villars ghost in a manner consistent with general covariance and achieving its renormalizability in an unphysical way. This interpretation of the classically scale invariant theory leaves room for doubt for several reasons. Perhaps it is simply the way in which mass was introduced that is at fault. The static potential associated with a $1 / q^{4}$ propagator is proportional to distance, $|\vec{x}|$, so, taken at face value, this would be a confining theory! The same conclusion is also suggested by the running of the gravitational couplings. The complement of their being asymptotically free (AF) is that they grow as the renormalization scale $\mu$ decreases, so one would expect the theory to become strongly coupled at lower energy scales. It seems unlikely that the resulting theory at large distances would look anything like general relativity, quite aside from whether or not it satisfies unitarity. Finally, the spectrum in a flat space background may not be relevant to theories having a curved spacetime background. It is notoriously difficult to determine the candidate no-particle states (or vacua) when gravity is included.

Another possibility is that the theory (without an explicit Einstein-Hilbert term) undergoes DT of the CW type discussed above, where gravitational couplings play the role of the electromagnetic coupling in scalar electrodynamics [3]. If that occurs and the couplings are weak, then it should be possible to infer the properties of the theory at that scale and of the effective field theory below. We shall show that, assuming maximally symmetric spacetime, DT can occur for weak couplings in $R^{2}$-gravity (eq. (1.1)), but the extrema are not locally stable and cannot be assumed to be the true no-particle or vacuum state.

If this theory is to look at all like Einstein gravity at low energies, it seems to be necessary to include matter. In order for $R^{2}$-gravity to remain natural, the matter field action must not only describe a renormalizable theory but also be classically scale invariant. This is automatically true for gauge bosons, but it is a strong constraint on scalars and fermions. Previous such attempts have been plagued by an effective action that contains an imaginary part, (reviewed, e.g., in ref. [19],) suggesting that such models become unstable. We shall see that is not the case here.

The simplest form of matter would be to add a real scalar field to eq. (1.1) in a classically scale-invariant manner

$$
S_{m}=\int d^{4} x \sqrt{g}\left[\frac{1}{2}(\nabla \phi)^{2}+\frac{\lambda}{4} \phi^{4}-\frac{\xi \phi^{2}}{2} R\right] .
$$

The non-minimal coupling $\xi$ is required for renormalizability. Perturbatively, of course, one might expect $\langle\phi\rangle=0$, as in the purely scalar theory. However, if $R^{2}$-gravity plays the role of electrodynamics in the Coleman-Weinberg model, DT may occur for some relation 
among the various couplings. If so, and $\langle\phi\rangle=v \neq 0$, then $8 \pi \xi v^{2} \equiv M_{P}^{2}$ would correspond to the Planck mass $M_{P}$ (assuming that $\xi>0$ ). Below this scale, the theory would look very much like ordinary general relativity. We shall show that DT can in fact occur and that the effective action in this model does not have an imaginary part. We find that this model has several fixed points, one of which is indeed AF. Unfortunately, even though the gravitational couplings $a, b$ are $\mathrm{AF}$, the basin of attraction of this $\mathrm{AF}$ fixed point does not include the range of matter couplings at which these minima occur. (One may hope that this disappointing result is model dependent and that more realistic models including, e.g., non-Abelian gauge fields and fermions, might not encounter such an obstruction). Nevertheless, thinking of $S_{\mathrm{ho}}+S_{m}$ as an effective field theory, these minima are candidates for stable vacua at the Planck mass scale and below. Whether they are also unitary theories has not been determined, although we shall discuss the issue further.

The outline of this paper is as follows: in the next section, we discuss the theory defined by the action eq. (1.1) and its renormalization in terms of essentially two coupling constants. In connection with this we explain the role of the Gauss-Bonnet term, and remark on the relationship of its renormalization and a possible $a$-theorem. Then, in section 3, we describe the nature of scale symmetry breaking in QFT and its implications for naturalness. In section 4 , we review the effective action in $R^{2}$-gravity, including the one-loop beta-functions for the couplings and their asymptotic freedom (AF). We show that the beta-functions for the couplings determine the form of the one-loop, $O(\hbar)$, correction to the effective action and investigate the possibility of DT at its extrema. We derive a (new) formula for the local curvature in order to determine whether an extremum is a (local) maximum, minimum, or saddle-point. An interesting aspect of this development is that, even though the curvature is $O\left(\hbar^{2}\right)$, it is determined entirely by the one-loop corrections. In section 5, we extend this formalism to the model with a massless, real scalar field, showing how DT may arise and discuss the low-energy effective field theory. Although we use the Jordan frame for the most part, we also discuss this model from the point of view of the Einstein frame. In section 6, we briefly discuss extending the model to include the Standard Model fields. In section 7, we discuss constraints on the coupling constants in order to expect theories of this sort to make sense both at the highest possible scales as well as at and below the DT scale. Finally, in section 8, we conclude with a discuss of open questions and future applications. In five appendices, we review some topics that bear on our work in an effort to make this paper more self-contained, viz., the Gauss-Bonnet relation, the background field method, the definition of scale invariance, constraints on the couplings required for stability, and the one-loop beta functions for models of this type.

\section{The action for pure $R^{2}$ "gravity"}

Because we are interested in classically ${ }^{3}$ scale invariant theories in four dimensions, the action for pure gravity will contain the quadratic invariants given in eq. (1.1) However, this is not the action that has been the starting point for analyses of this theory [1-4]. This is

\footnotetext{
${ }^{3}$ By "classical," we simply mean the tree approximation in terms of renormalized couplings and fields associated with some conveniently chosen scale.
} 
because of the Gauss-Bonnet relation, which in differential form may be expressed as

$$
R^{*} R^{*}=C_{\kappa \lambda \mu \nu}^{2}-2 \widehat{R}_{\mu \nu}^{2}+\frac{1}{6} R^{2} \equiv G
$$

where $\widehat{R}_{\mu \nu} \equiv R_{\mu \nu}-g_{\mu \nu} R / 4$ is the traceless Ricci tensor. The properties of the "topological" term $R^{*} R^{*}$ and its relation in integral form to the Euler characteristic are summarized in appendix A. For our purposes, it is sufficient to know that it can be written as the divergence of a current, $R^{*} R^{*}=\nabla_{\mu} B^{\mu}$. As a result, the variation of its contribution to the action vanishes identically

$$
\frac{\delta}{\delta g_{\mu \nu}} \int d^{4} x \sqrt{g} G=0 .
$$

This property is closely related to the validity of the Bianchi identities. Although special to four dimensions, these act like another symmetry that reduces the number of independent couplings. The action eq. (1.1) can be rewritten, for example, as

$$
S_{\mathrm{ho}}=\int d^{4} x \sqrt{g}\left[\frac{1}{2 a} C_{\kappa \lambda \mu \nu}^{2}+\frac{1}{3 b} R^{2}+\varepsilon G\right] .
$$

According to eq. (2.2), the last term contributes nothing to the variation of the action, so one might think it could be discarded altogether. When formulating the Feynman rules in four-dimensions, it is certainly irrelevant so that, in fact, this theory would appear to be renormalizable in terms of two coupling constants only $(a, b)$. However, the theory without the $\varepsilon G$ term is not multiplicatively renormalizable. ${ }^{4}$ Because of its relative simplicity and manifest gauge invariance, the regularization scheme usually chosen is dimensional regularization (DREG). This confuses the issue further because, for dimension $n \neq 4$, the operator $\sqrt{g} G$ cannot be expressed as a total derivative, nor can any dimension-dependent linear combination of the three renormalized operators [20]. One might be tempted to conclude that, like scale invariance or chiral symmetry, the Gauss-Bonnet relation was anomalous or at least inconsistent with DREG [21]. Fortunately, it is enough to extend $\sqrt{g}\left(C_{\kappa \lambda \mu \nu}^{2}-2 \widehat{R}_{\mu \nu}^{2}+R^{2} / 6\right)$ to $n$-dimensions, which is possible. Any definition for continuous $n$ that reduces to this linear combination as $n \rightarrow 4$ should suffice. This enables the definition of renormalized operators and couplings in four dimensions, at which point, one may then rewrite $G=R^{*} R^{*}=\nabla_{\mu} B^{\mu}$ locally, using the special properties of the curvature tensor in four dimensions, such as the Bianchi identities.

Nevertheless, the extension of eq. (2.2) to $n$-dimensions will not be correct, so one would think that one needs to include $G$ in constructing the Feynman rules in $n$-dimensions, adding further complications to renormalization of the theory. In fact, this obstacle has been circumvented by previous authors [1-4]. In practice, this has been accomplished as follows: ignoring $\varepsilon G$ when determining the Feynman rules, one finds that the theory is not multiplicatively renormalizable unless one includes counterterms for $\varepsilon G$ as well. Since

\footnotetext{
${ }^{4}$ This has nothing to do with the regularization chosen. It is the fact that there are three independent scalar quadratic invariants, given in eq. (1.1), and divergences occur proportional to each of them. It would be equally true using a regularization scheme operating within four-dimensions. This is discussed further below and in appendix B.
} 
these divergences (up to finite local counterterms) determine the beta-functions, this would imply that $\beta_{\varepsilon}$ is a function only of the remaining couplings, $a, b$. If so, then it must be the case that

$$
\beta_{\varepsilon}=\frac{\partial \varepsilon}{\partial a} \beta_{a}(a, b)+\frac{\partial \varepsilon}{\partial b} \beta_{b}(a, b)
$$

to all orders in perturbation theory. This is a nontrivial statement about the renormalized couplings in four-dimensions. Among other things, it implies that there must then be a tree-level contribution to $\varepsilon(a, b)$ as well. In this theory, because eq. (2.2) is correct in four dimensions, it is possible to solve eq. (2.4) order-by-order in perturbation theory. In a separate publication [22], we prove this is possible and determine the function $\varepsilon(a, b)$ in lowest order to be $\varepsilon=\varepsilon_{0}-\beta_{1} /\left(\beta_{2} a\right)$, where $\varepsilon_{0}$ is a scale-independent constant. ( $\beta_{1}$ and $\beta_{2}$ are constants entering the one loop beta-functions $\beta_{\varepsilon}$ and $\beta_{a}$, respectively, given below and in appendix E.)

We should emphasize that eq. (2.4) and the remarks below it apply only in the model without matter fields. In general, we would expect $\beta_{\varepsilon}$ to be a function of all the other dimensionless coupling constants in the theory, except, as we have described, $\varepsilon$ itself. Indeed, $\beta_{\varepsilon}$ is nonzero even if we do not quantize gravity, in other words there are "pure matter" contributions, independent of $a, b$. One might expect such contributions to appear at two loops from graphs with two gauge couplings or two Yukawa couplings, and at three loops from graphs with two quartic scalar couplings. One sees, however, from ref. [23] and ref. [24], that although such graphs generate contributions to $\beta_{a}$ and $\beta_{b}$, they do not contribute to $\beta_{\varepsilon}$.

In fact, $\beta_{\varepsilon}$ as described here is the Euler anomaly coefficient, that is, the coefficient of $G$ in the gravitational trace anomaly. It thus represents a generalization to the quantized $R^{2}$-gravity case of the candidate $a$-function proposed by Cardy [25] as manifesting a 4dimensional $c$-theorem. Results for this anomaly coefficient (without quantizing gravity) include a non-zero 5-loop contribution involving four quartic scalar couplings [26] and nonzero three loop contributions involving gauge and Yukawa couplings [27]. For more on the $a$-theorem see [28-30]; for some recent progress see [31, 32], and, for some interesting potential cosmological consequences, see [33].

One consequence of our considerations is that, even though $G$ is a covariant divergence and $\sqrt{g} G$ is an ordinary derivative, it can contribute a nonzero value to the action in eq. (2.3) in curved spacetime just like the other terms, even though it is equivalent to a "surface" term or "boundary" term. For example, in a maximally symmetric background, $G=R^{2} / 6$. It is paradoxical that a "surface term" could be of the same order as a volume term in the action. Even more, Euclidean de Sitter space is topologically the sphere $S^{4}$, so that there is no boundary or surface whatsoever, yet the integral is nonzero, apparently violating Gauss's law. The resolution of this paradox is that although $G=\nabla_{\mu} B^{\mu}$ is gauge-invariant, $B^{\mu}$ is not, i.e., it does not transform as a vector under general coordinate transformations. ${ }^{5}$ This is related to the fact that the surface $S^{4}$ is homotopically nontrivial.

\footnotetext{
${ }^{5}$ In other words, $\sqrt{g} G$ is closed but not exact on $S^{4}$. See appendix A.
} 


\section{$3 \quad$ Scale symmetry breaking and naturalness}

This theory is classically scale-invariant but not conformally invariant. The associated QFT breaks scale invariance through the renormalization procedure by which the coupling constants become scale-dependent. Classical scale symmetry is therefore anomalous in QFT; the divergence of the dilatation current, instead of vanishing, becomes the sum of beta-functions of couplings or masses times their corresponding operators.

This anomaly has nothing to do with naturalness [12], which is associated with powerlaw divergences, typically characterized in terms of some cutoff $\Lambda$ as quadratic behaviour $\Lambda^{2}$ for scalar masses or $\Lambda^{4}$ for the vacuum energy, times some coupling constants. This is a physical effect perhaps best illustrated in the context of grand unified theories (GUTs) in which the $\mathrm{SU}(2) \otimes \mathrm{U}(1)$ electroweak theory is embedded in some larger group $G$, such as $\mathrm{SU}(5)$. The GUT theory involves particle masses $M_{U} \gg M_{W}$, and it is difficult to arrange for the ratio $M_{W} / M_{U}$ to be as small as required, $10^{-13}-10^{-14}$, because radiative corrections to the lighter masses such as $M_{W}$ are often proportional to the larger scale $M_{U}$. This provides motivation for softly broken supersymmetry (susy), still the most popular extension of the Standard Model (SM). Any theory in which such effects are suppressed seems to depend upon some symmetry to protect it.

Classically scale invariant theories, although anomalous, beget a legacy to their corresponding QFT's. As has been emphasized by Bardeen [7, 8] and others [9], the breaking of scale invariance by anomalies is "soft", reflecting logarithmic divergences of the "bare" theory that are responsible for running couplings. This is not true for power divergences, a radiative correction behaving, for example, as $g^{2}(\Lambda) \Lambda^{2} /(4 \pi)^{2}$. Even if the coupling $g^{2}(\Lambda)$ were $\mathrm{AF}$, it would vanish relatively slowly, as $1 / \log (\Lambda)$ as $\Lambda \rightarrow \infty$, so that $g^{2}(\Lambda) \Lambda^{2}$ does not become small. Power-law divergences are therefore incompatible with a theory having classical scale invariance. Turning this around, this is why effective field theories that are intended to apply below some physically relevant higher mass scale are not classically scale invariant. Such models usually have radiative corrections that behave like powers of the high scale. In the present circumstances, in which we wish to entertain the possibility that there are no physically relevant higher mass scales, it is perfectly natural to ignore potential power divergences as manifestations of the regularization method. In fact, DREG is a regularization procedure that does assign the value zero to power divergences, which is the correct procedure in the present context.

As mentioned above, not only is the pure gravity theory AF, but it conveys this property to the dimensionless matter couplings $[2,6]$ that may be added, so that the ultraviolet behaviour for many of these models is perfectly natural. ${ }^{6}$

Previous workers have added an Einstein-Hilbert term $M_{P}^{2} R$ and a cosmological constant $\Lambda_{c c}$, thereby explicitly breaking classical scale invariance. This theory remains formally renormalizable and $\mathrm{AF}$, since $M_{P}^{2} R$ and $\Lambda_{c c}$ are UV irrelevant operators. From this point of view, this looks acceptable, and, assuming that the couplings $a, b$ are still sufficiently small on the scale $M_{P}$, the effective field theory below $M_{P}$ will look conventional.

\footnotetext{
${ }^{6} \mathrm{~A}$ word of caution must be issued here; in the case of a single real field, we find that the basin of attraction of the UV fixed point is limited.
} 
Flat spacetime would appear to be a sensible solution to eq. (4.2) at large distances, but it is easily seen that perturbations about that background have a massive spin-two field with negative kinetic energy. This is the origin of the belief that the theory violates unitarity. From another point of view, however, the addition of these irrelevant couplings to the bare theory is a drastic modification, since it is no longer natural to ignore power-law divergences associated with radiative corrections. As a result, it would appear to require extremely fine tuning to sustain this form of the theory, so it would be impossible to argue that it represents a UV completion of general relativity. Consequently, this theory is unacceptable as a starting point for a completion of gravity, and such a model must be interpreted as an ordinary effective field theory in which the terms quadratic or quartic in curvature are simply some of the operators that can be expected to become important at energy scales on the order of $M_{P}$ but small compared to some large physical cutoff $\Lambda_{\text {eff }}$.

In order to account for ordinary Einstein gravity in a natural way, models such as the ones considered herein, described by $S_{\text {ho }}$ plus matter, must undergo DT, as described in the Introduction, section 1. In the next section, we review and extend the formalism for investigating this possibility perturbatively.

\section{Dimensional transmutation in $R^{2}$ gravity}

The formalism will be reviewed for a case that has already been partially discussed in the literature $[2,5,6]$, although from a rather different perspective. For this purpose, it will be useful to define the rescaled coupling $w \equiv a / b$, so that the action eq. (2.3) becomes

$$
S_{\mathrm{ho}}=\int d^{4} x \sqrt{g}\left[\frac{1}{a}\left(\frac{1}{2} C_{\kappa \lambda \mu \nu}^{2}+\frac{w}{3} R^{2}\right)+\varepsilon G\right] .
$$

This form has several advantages. The AF coupling a may also be identified with the loop-expansion parameter, whereas the coupling $w$ will be seen to approach a UV fixed point. As we shall discuss below, the form of the $\beta$-functions suggest treating $a$ as the primary coupling governing the asymptotic behaviour of the others.

As usual, the investigation of spontaneous symmetry breaking (SSB) of a theory involves the effective action $\Gamma\left[g_{\mu \nu}(x)\right]$. Like the classical action, it is a functional of the fields. The extrema of the effective action determine candidates for local minima, maxima, and saddle-points:

$$
\frac{\delta}{\delta g_{\mu \nu}(x)} \Gamma\left[g_{\mu \nu}\right]=0
$$

Metrics satisfying this equation are said to be "on-shell". We have suppressed the dependence of $\Gamma\left[g_{\mu \nu}\right]$ upon the coupling constants $a(\mu), w(\mu), \varepsilon(\mu)$ and the normalization scale $\mu$, but they are important. The effective action obeys the renormalization group equation (RGE)

$$
\left[\mu \frac{\partial}{\partial \mu}+\beta_{a} \frac{\partial}{\partial a}+\beta_{w} \frac{\partial}{\partial w}+\beta_{\varepsilon} \frac{\partial}{\partial \varepsilon}+\beta_{\sigma_{j}} \frac{\partial}{\partial \sigma_{j}}-\gamma \int d^{4} x g_{\mu \nu}(x) \frac{\delta}{\delta g_{\mu \nu}(x)}\right] \Gamma\left[g_{\mu \nu}\right]=0,
$$

where $\sigma_{j}$ denote possible gauge-fixing parameters, and $\gamma$ the anomalous dimension of the metric. The effective action is the generator of the 1PI $n$-point functions 
$\Gamma_{n}\left(g_{\mu \nu}\left(x_{1}\right), g_{\mu \nu}\left(x_{2}\right), \ldots, g_{\mu \nu}\left(x_{n}\right)\right)$, and it is nonlocal in general. In perturbation theory, the "classical" action consists of a term of the form of eq. (4.1). Radiative corrections consist of loop diagrams plus divergent counterterms of the same form as eq. (4.1) such that all $\Gamma_{n}\left[g_{\mu \nu}\left(x_{j}\right)\right]$ remain finite as the cutoff is removed.

The one-loop effective action has not been determined for an arbitrary background metric, so it is impossible to discuss all possible solutions of eq. (4.2). However, it is clear that, formally, this equation will have a solution for flat spacetime, $g_{\mu \nu}=\eta_{\mu \nu}$, where all curvature tensors vanish. However, as we have remarked, we do not expect this to be a consistent background solution of the QFT, because the couplings become strong in the infrared, and this appears to be a confining theory. In this respect, it is similar to YangMills theory. Just what a consistent solution looks like, we do not know, but we would expect the emergence of a DT scale, $\Lambda_{\text {ho }}$. Since the theory has more than one coupling, there may remain free parameters on which the spectrum and interactions could depend. Whether there can be any light states below $\Lambda_{\text {ho }}$ is unclear, but, regardless, it is highly unlikely that this theory would resemble conventional gravity at long distances.

Since the original theory eq. (4.1) has more than one coupling constant, one may ask whether DT can occur for weak coupling. To our knowledge, this has not been explored before. To simplify the analysis, we shall assume that the field is maximally symmetric, so that the metric describes either de Sitter (dS) or anti-de Sitter (AdS) spacetime, depending on whether the constant curvature is positive or negative. We shall investigate whether DT can occur for a particular value of the curvature $R$. For the dS case, the Euclidean manifold is usually compactified on a four-sphere [5] because the isometries of $\mathrm{dS}$ are the rotations $\mathrm{SO}(5)$ (or $\mathrm{SO}(1,4)$ for Lorentzian signature.) The global topology is unimportant in perturbation theory. For Euclidean AdS, the isometry group is $\mathrm{SO}(1,4)$ for Euclidean signature (or $\mathrm{SO}(2,3)$ for Lorentzian signature). In this case, the associated spacetime is hyperbolic, so the manifold inherently has infinite volume. In either case, the maximallysymmetric background has $C_{\kappa \lambda \mu \nu}=0, R_{\mu \nu}=g_{\mu \nu} R / 4$. Therefore, $G=R^{2} / 6$, and the value of the classical action is

$$
S_{\mathrm{ho}}=\int d^{4} x \sqrt{g} \frac{R^{2}}{3}\left(\frac{1}{b}+\frac{\varepsilon}{2}\right) .
$$

All we really need to know about the volume element is that $d^{4} x \sqrt{g} \propto 1 / R^{2}$, which can be inferred from dimensional analysis alone. Thus,

$$
\int d^{4} x \sqrt{g} \equiv \frac{V_{4}}{R^{2}}, \quad \text { so } S_{\mathrm{ho}}=V_{4}\left(\frac{1}{3 b}+\frac{\varepsilon}{6}\right)=\frac{V_{4}}{6}\left(\frac{2 w}{a}+\varepsilon\right),
$$

where $V_{4}$ is some dimensionless volume element independent of $R$. For the four-sphere of $\mathrm{dS}, V_{4}=6(8 \pi)^{2}$, while for (the cover of) hyperbolic AdS, it is infinite, so we have to imagine a temporary large distance cutoff so that the spacetime has a finite volume. Alternatively, we can isolate the reduced effective action $S_{\text {ho }} / V_{4}$, which is the analog of the effective potential in flat spacetime.

Consider the calculation of the effective action $\Gamma\left[g_{\mu \nu}^{B}\right]$ by the background field method, ${ }^{7}$ which involves shifting the metric $g_{\mu \nu}=g_{\mu \nu}^{B}+h_{\mu \nu}$ by a classical field and treating

\footnotetext{
${ }^{7}$ The background field method is reviewed briefly in appendix B.
} 
$h_{\mu \nu}$ as the quantum field. ${ }^{8}$ The background field in our case will be assumed to have maximal symmetry, but the quantum field over which we integrate is arbitrary. In order that fluctuations about the background be stable, there may be restrictions on the coupling constants. For example, Avramidi [5] showed that the couplings must obey the constraints, e.g., $a(\mu)>0,0<w(\mu)<3 / 2$, for convergence of the Euclidean path integral, but he did not indicate at what scale $\mu$ such inequalities must hold. We shall return to these issues in section 7; see also appendix D for further details.

For a maximally symmetric background, the only unknown quantity is the magnitude of the curvature $R$. In this paper, we shall only investigate in detail the case of positive curvature, leaving AdS for later work. Let us call $\rho \equiv \sqrt{R}$. The question is whether $\rho$ may be determined by DT. The effective action $\Gamma$ can depend only on $\rho, \mu, a(\mu), w(\mu), \varepsilon(\mu)$. In fact, given eq. (2.2), only the "classical" action can depend on the parameter $\varepsilon$. Therefore, it will not enter the Feynman rules for calculating radiative corrections $\Delta \Gamma$ to the effective action. Nevertheless, $\varepsilon$ is renormalized and does require counterterms which, however, only depend on the other coupling constants. (For further discussion, see appendix B.)

Since $\Gamma$ is dimensionless, its scale dependence must be in terms of the ratio $\rho / \mu$. We may therefore express its loop expansion in the following form: ${ }^{9}$

$$
\Gamma(\rho)=S_{\mathrm{ho}}(a, w, \varepsilon)+B(a, w) \log (\rho / \mu)+\frac{C(a, w)}{2} \log ^{2}(\rho / \mu)+\frac{D(a, w)}{6} \log ^{3}(\rho / \mu)+\ldots
$$

The coefficients $B, C, \ldots$ are functions of the dimensionless couplings $(a(\mu), w(\mu))$, but the dependence on $\log \mu$ has been exhibited explicitly. In the loop expansion,

$$
B(a, w) \equiv \sum_{1}^{\infty} B_{k}(w) a^{k-1}, C(a, w) \equiv \sum_{2}^{\infty} C_{k}(w) a^{k-1}, D(a, w) \equiv \sum_{3}^{\infty} D_{k}(w) a^{k-1}, \ldots,
$$

where the coefficients $B_{k}, C_{k}, D_{k}$, etc., represent the contribution $k$-th order. In general, the coefficient of the power $\log ^{n}(\rho / \mu)$ is nonzero beginning at loop-order $k=n$. At oneloop, only the term with coefficient $B$ arises; at two-loops, the term having coefficient $C$ also arises, etc. The first derivative of the effective action is

$$
\frac{\partial \Gamma}{\partial \rho}=\frac{1}{\rho}\left[B(a, w)+C(a, w) \log (\rho / \mu)+\frac{D}{2} \log ^{2}(\rho / \mu)+\ldots\right] .
$$

An extremum at $\rho=v \neq 0$ satisfies eq. (4.2), which in the present application, reduces to an ordinary derivative, $\Gamma^{\prime}(v)=0$ in eq. (4.8). Obviously, this equation simplifies considerably if we choose to normalize at $\mu=v$ :

$$
\left.\frac{\partial \Gamma}{\partial \rho}\right|_{\rho=v}=\frac{1}{v}[B(a(v), w(v))]=0 .
$$

\footnotetext{
${ }^{8}$ Alternate definitions of the quantum field are sometimes used. See ref. [6] for further discussion.

${ }^{9}$ In general, there will also be a term $A(a, w)$ on the right-hand side representing finite local counterterms characteristic of the particular renormalization scheme. Even for minimal subtraction (MS), it is nonzero. We shall assume that the renormalization prescription has been modified in such a way as to remove such terms, which, while they could be included, only serve to complicate our subsequent discussion. See below, however, concerning the possibility of an imaginary part of $\Gamma$.
} 
The meaning of this stark equation is that, given the function $B(a, w)$, an extremum will occur if one can find a scale $v$ at which the couplings are related according to the equation $B(a(v), w(v))=0$. At one-loop order, this corresponds to a value of the coupling $w=w_{1}$, where $B_{1}\left(w_{1}(v)\right)=0$, independent of $a$ !

To characterize this extremum as a local maximum or minimum, we must know

$$
\delta^{(2)} \Gamma=\frac{1}{2} \Gamma^{\prime \prime}(v)(\delta \rho)^{2}=\frac{1}{2 v^{2}} C(a(v), w(v))(\delta \rho)^{2},
$$

where $C$, we recall from eq. (4.7), starts at two-loop order $C_{2}(w) a$. This is in fact the mass of the dilaton which arises from the scale-breaking anomaly. In fact, we shall see that $C_{2}$ can be determined from one-loop results. Because we have assumed such a simple background, one can determine the form ${ }^{10}$ of $B_{1}(w)$ and $C_{2}(w)$ directly using the RGE, eq. (4.3), which we write in the form

$$
-\left[\mu \frac{\partial}{\partial \mu}-\gamma \rho \frac{\partial}{\partial \rho}\right] \Gamma(\rho)=\left[\beta_{a} \frac{\partial}{\partial a}+\beta_{w} \frac{\partial}{\partial w}+\beta_{\varepsilon} \frac{\partial}{\partial \varepsilon}\right] \Gamma(\rho)+\ldots
$$

Using the fact that $\Gamma(\rho)$ depends on $\rho$ only through the ratio $\rho / \mu$, the left-hand side may be written as $(1+\gamma) \rho \partial \Gamma / \partial \rho$. The only dependence on $\varepsilon$ is through the "classical" action, eq. (4.1), (including counterterms), and $\beta_{\varepsilon}$ is related to the other beta-functions through eq. (2.4).

We have suppressed the gauge-dependent terms on the right-hand side of eq. (4.11), as they will not affect our results. We will find that the RG equation relates $B(a, w)$, $C(a, w)$ etc to the $\beta$-functions and the gauge-dependent anomalous dimension $\gamma$. However the dependence on $\gamma$ cancels out in on-shell (i.e. physical) quantities. In the case involving a matter field, to which we will turn in the next section, this cancellation is quite nontrivial because (as we shall see) in that case both $B_{1}$ and $C_{2}$ depend on $\gamma$ in general.

It was observed long ago that the RGE relates different orders of the loop-expansion for $\Gamma$ [34]. The beta-functions and anomalous dimensions have loop expansions of the same form as $B(a, w)$ in eq. (4.7), so, if they are known to some order, then one may insert them into the RGE eq. (4.3), together with the loop expansion in eq. (4.6), and equate common powers in $a^{n}$ (or $\hbar^{n}$ ). Thus (inserting explicit factors of $\hbar$ for clarity) we find

$$
\begin{aligned}
\mu \frac{\partial \Gamma}{\partial \mu} & =-\hbar B_{1}-\hbar^{2} B_{2}-\hbar^{2} C_{2} \ln (\rho / \mu)+\cdots \\
\sum_{i} \beta_{i} \frac{\partial \Gamma}{\partial \lambda_{i}} & =\sum_{i}\left(\hbar \beta_{i}^{(1)}+\hbar^{2} \beta^{(2)}\right) \frac{\partial}{\partial \lambda_{i}} S_{\mathrm{ho}}+\hbar^{2} \sum_{i} \beta_{i}^{(1)} \frac{\partial}{\partial \lambda_{i}} B_{1} \ln (\rho / \mu)+\cdots \\
-\gamma \rho \frac{\partial}{\partial \rho} \Gamma & =-\hbar^{2} \gamma^{(1)} B_{1}+\cdots
\end{aligned}
$$

where $\beta_{i}^{(1)}$ denotes the one-loop beta-function for the coupling $\lambda_{i}, S_{\mathrm{ho}}$ is given in eq. (4.5), and the sums are over all couplings on which the classical action depends $\{a, w, \varepsilon\}$. It

\footnotetext{
${ }^{10}$ In ref. [5], Avramidi calculated $B_{1}$ by explicitly evaluating the functional determinants arising at oneloop. See appendix B for further discussion. He checked his result by showing that it satisfied the RGE. Our result for $C_{2}$ is new.
} 
follows that

$$
\begin{aligned}
B_{1} & =\sum_{i} \beta_{i}^{(1)} \frac{\partial}{\partial \lambda_{i}} S_{\mathrm{ho}} \\
a C_{2} & =\sum_{i} \beta_{i}^{(1)} \frac{\partial}{\partial \lambda_{i}} B_{1}=\left[\sum_{i} \beta_{i}^{(1)} \frac{\partial}{\partial \lambda_{i}}\right]^{2} S_{\mathrm{ho}}, \\
a B_{2} & =\beta^{(2)} \frac{\partial}{\partial \lambda_{i}} S_{\mathrm{ho}}-\gamma^{(1)} B_{1} .
\end{aligned}
$$

Thus, from eqs. (4.15), (4.16), we obtain the leading contributions to both the condition for an extremum (eq. (4.9)) and its nature (eq. (4.10)). ${ }^{11}$ Note that neither condition depends on the anomalous dimension $\gamma$.

In MS, in each order of the loop expansion, the only really new contribution is to the single $\log$ term, $B$, with all the higher powers of $\log (\mu)$ determined by lower-order corrections. ${ }^{12}$

There is a possible flaw in the preceding method of determining the effective action. Although, as mentioned in an earlier footnote, it is possible to adopt a renormalization prescription to remove real local counterterms $A(a, w)$, if the effective action had an imaginary part of this form, it could not removed by counterterms. If present, an imaginary part must be regarded as an instability. In a direct evaluation of the functional determinants, it would show up as a negative eigenvalue that would prevent one from carrying out the path integral. Just as in flat space models, such as scalar $\lambda \phi^{4}$ theory with a negative $m^{2} \phi^{2}$ term, such a term could arise by continuation of the effective potential from a region where there is no imaginary part to another region where the argument of a logarithm turns negative. ${ }^{13}$ Another potential shortcoming of this method is that it does not reveal whether there are zero modes. In fact, as shown in [5], there are five in the conformal sector of the metric fluctuations.

Of course, once one has a formula for the one-loop corrections to the real part of the effective action via the RGE, one can check whether or not fluctuations are unstable and whether there remain flat directions, and this can be done without performing any functional integrations. We shall discuss this further in section 7 .

To apply these formulas, we need the one-loop beta-functions [4];

$$
\begin{array}{ll}
\frac{1}{\kappa} \beta_{a}^{\text {ho }}=-\beta_{2}^{\text {ho }} a^{2}, \quad \beta_{2}^{\text {ho }}=\frac{133}{10}, \quad \frac{1}{\kappa} \beta_{\varepsilon}^{\text {ho }}=-\beta_{1}^{\text {ho }}, \beta_{1}^{\text {ho }}=\frac{196}{45}, \\
\frac{1}{\kappa} \beta_{w}^{\text {ho }}=\frac{10 a}{3}\left[w^{2}-\frac{549}{100} w+\frac{1}{8}\right],
\end{array}
$$

\footnotetext{
${ }^{11}$ One can check that the results in eqs. (4.15), (4.16) are unchanged by the addition of finite local counterterms $A_{0}(a, w)$. That will not be true for the two-loop contributions to $B$, for example.

${ }^{12}$ One can check that the results in eqs. (4.15), (4.16) are unchanged by the addition of finite local counterterms $A_{0}(a, w)$. That will not be true for the two-loop contributions to $B$, for example.

${ }^{13}$ Avramidi [5] actually did evaluate the functional determinants for a similar model that included an Einstein-Hilbert term as well as a cosmological constant. We can take advantage of his calculation to check some of our results, but he did not evaluate the curvature $C_{2}$. One can in principle obtain the results below from his by forming the RG-improved effective action starting from his one-loop effective action.
} 
where $1 / \kappa \equiv 16 \pi^{2}$. For $a>0, \beta_{a}$ displays AF, as claimed, and the sign of $a$ is renormalization group $(\mathrm{RG})$ invariant. In order to have a Euclidean action bounded from below, we require $a>0$. The running of $w$ is more complicated; $\beta_{w}$ has two real zeros. There is a UV fixed point at $w_{1} \approx 0.023,(a \ll b)$, and an IR fixed point at $w_{2} \approx 5.47(a>b)$. Naively, it appears as if $w=0$ is neither a singular point nor a fixed point, but $1 / w=b / a \rightarrow \infty$ as $w(\mu) \rightarrow 0$, and therefore $b \rightarrow \infty$ (a "Landau" pole). Since perturbative corrections are polynomials in the parameters $(a, b)$, this constitutes a breakdown of perturbation theory. Typically, we expect perturbation theory to be valid only for $\kappa a \ll 1$ and $\kappa b \ll 1$, so we cannot trust the one-loop results arbitrarily near $w=0$.

On the other hand, $w \rightarrow \infty$ corresponds to $b \rightarrow 0$, which is not a breakdown of perturbation theory. It would have been better to take the ratio of couplings as $\widetilde{w}=$ $1 / w=b / a$, since perturbation theory holds as $\widetilde{w} \rightarrow 0$, but we shall continue to follow past conventions.

To determine possible extrema perturbatively, we may evaluate eqs. (4.15), (4.16) in the one-loop approximation, yielding:

$$
\begin{aligned}
& B_{1}=V_{4} \frac{10 \kappa}{9}\left[w^{2}-\frac{3}{2} w-\frac{317}{600}\right], \\
& C_{2}=V_{4} \beta_{w} \frac{20 \kappa}{9}\left(w-\frac{3}{4}\right)=V_{4} a \frac{200 \kappa^{2}}{27}\left[w^{2}-\frac{549}{100} w+\frac{1}{8}\right]\left(w-\frac{3}{4}\right) .
\end{aligned}
$$

The extrema occur where $B_{1}=0$, viz., $w_{ \pm}=3 / 4 \pm \sqrt{3927} / 60$. For both the positive root, $w_{+} \approx 1.794$ and the negative root $w_{-} \approx-0.294$, we find from eq. (4.20) that $C_{2}<0$, and hence (from eq. (4.10)) that both extrema are local maxima of the action.

Even though they are not locally stable, we would like to determine whether these extrema can be reached naturally in the course of the running of coupling constants or whether fine-tuning would be required to arrange for these values of the coupling constants. This may be less interesting than if they were minima, but the analysis serves to illustrate concepts useful in models having additional coupling constants with more complicated renormalization flows. Further, as we shall discuss, it is conceivable that maxima such as these and saddle points could be cosmologically relevant.

To discuss the running of the couplings, we shall assume that the initial values $a_{0}, b_{0}$ are sufficiently small so that perturbation theory may be used at the starting point. In view of the fixed points at $w=w_{1}$ and $w=w_{2}$, there are three possible phases to be discussed: (1) $w_{1}<w(\mu)<w_{2},(2) w(\mu)>w_{2}$ or $w(\mu)<0$, and $(3) 0<w(\mu)<w_{1}$. As noted, we would expect perturbation theory to be valid so long as $\kappa a(\mu) \ll 1$ and $\kappa b(\mu) \ll 1$, and any initial value of the ratio $w_{0}=a_{0} / b_{0}$ can be accommodated perturbatively except for $w \rightarrow 0$, where $b \rightarrow \infty$.

1. Starting at any value $w_{0}$ between the two fixed points, $w_{1}<w_{0}<w_{2}, w(\mu)$ spans the entire region by running toward higher or lower scales $\mu$. The extremum corresponding to $w=w_{+} \approx 1.79$ lies within this region and will be accessible perturbatively at some scale $v$, so long as $\kappa a(v) \ll 1$. Perturbation theory certainly holds in a neighborhood of $w_{1} \approx 0.023$, even though $b \gg a$, since both $a(\mu)$ and $b(\mu)$ vanish as $\mu \rightarrow \infty$. 
Perturbation theory will break down as $\mu$ decreases, since $a(\mu)$ monotonically increases. Although formally $w(\mu) \rightarrow w_{2}$ as $\mu \rightarrow 0$, the theory will become strongly coupled at some finite value of $\mu$.

2. Starting at any $w_{0}$ for $w_{0}>w_{2}$, we see that $w(\mu) \rightarrow+\infty$ as the scale $\mu$ increases. Since $a(\mu), b(\mu)$ are decreasing, this does not constitute a breakdown of perturbation theory, but it simply means that $b(\mu)$ passes through zero at some finite value of $\mu$. Since $b=0$ is not a fixed point, as $\mu$ increases further, $b(\mu)$ turns negative, and therefore also $w(\mu)<0$. $w(\mu)$ continues increasing through negative values toward the extremum at $w=w_{-} \approx-0.29$. So long as $|b|$ does not become too large, this could remain within the reach of perturbation theory. A similar story obviously holds if the starting value is in the region $w_{0}<w_{-}$. The couplings are continuous at $w=\infty$, so this point should be thought of as compactified.

If the initial value $w_{-}<w_{0}<0$, then one must decrease the scale $\mu$ to run toward $w_{-}$. Whether the DT scale $v$ can be reached will depend on whether perturbation theory continues to hold as $a$ increases and $|b|$ decreases.

3. With $0<w_{0}<w_{1} \approx+.023$, there is no extremum of the action in this region, so the behavior of the couplings is irrelevant for DT in this "pure gravity" model. Nevertheless, for completeness, we shall remark on the running. As the scale increases, $w \rightarrow w_{1}$, and $a$ and $b$ are AF. Decreasing the scale runs toward the scale where $b \rightarrow+\infty$, and perturbation theory breaks down. We guess this would occur for $\kappa b(\mu) \sim 1$. To be slightly more quantitative, if the initial value $\kappa b_{0} \sim 1$, then $\kappa a_{0} \sim w_{0}<w_{1}$, or $a_{0}<w_{1} / \kappa \approx 3.61$. The range of validity of perturbation theory therefore depends on how much smaller $a_{0}$ is than this.

In summary, we have found that there are two extrema at scales $\mu=v$ determined, for $b(v)>0$ by $a(v) / b(v)=w_{+} \approx 1.79$ and another, for $b(v)<0$, by $a(v) / b(v)=w_{-} \approx-0.294$. Both can be reached in perturbation theory starting from a wide range of initial values; however, both are local maxima since $C_{2}<0$, i.e., the dilaton is tachyonic. By our method of calculation, we cannot tell whether these extrema occur for $R>0$ (de Sitterlike) or $R<0$ (anti-de Sitter-like), but there is good reason to presume that it is valid in de Sitter background.

Since these are metastable vacua in de Sitter background, they might be candidates for "new inflation" scenarios if the local maxima are sufficiently flat. A quantitative measure of the degree of flatness in conventional models is the slow-roll parameter $\eta=M_{P}^{2} V^{\prime \prime}(\phi) / V(\phi)$, where $V(\phi)$ is the potential at the field value of interest. By transforming to Einstein frame, one can show that the corresponding quantity in our model is $\eta=m_{d}^{2} / \Lambda$, where $m_{d}$ is the dilaton mass proportional to $C_{2}$, and $\Lambda$ is the corresponding cosmological constant. Having determined $R=v^{2}$ from $B_{1}(w)=0$, we find that $\eta=2 C_{2} v^{2} /\left(3 M_{P}^{2}\right)$, where $C_{2}$ is given in eq. (4.20). (The appearance of $M_{P}$ here is due to the fact that in the Einstein frame the theory takes the form of Einstein gravity with a positive cosmological constant, coupled to a massless scalar.) As discussed earlier, the dilaton mass arises from the scale anomaly at two-loop order, so with reasonable values of the couplings, one can expect $\eta$ to be small. 
We have not analyzed this model at finite temperature, and we do not know the limit on $a(v)$ that would allow sufficient inflation. ${ }^{14}$

Although this is not a realistic model of our universe, this is a rather different inflationary mechanism than has been encountered previously. The metric is in a sense self-inflating. Of course, unlike Einstein-Hilbert theory, the metric in this model has additional degrees of freedom beyond the massless graviton, including a scalar mode, but it is not obvious that that this mode may be identified as the inflaton. Nevertheless, without any fine tuning, this already has some of the ingredients of a successful inflationary model, except, of course, that it is unlikely to exit to a phase that resembles general relativity, a problem that may be cured with the introduction of matter.

\section{Matter: the real scalar field}

In order to obtain a realistic field theory of gravity, it seems necessary to include matter fields. We shall simply discuss a real scalar field here, leaving the addition of other scalars, gauge fields and fermions for later work. The hope is that the matter action eq. (1.2) will lead to a nonzero vacuum expectation value for $\phi$, so that we may identify $\xi \phi^{2}$ with the reduced Planck scale $M_{P}^{2} / 8 \pi$. The idea of generating the Planck mass in this way is not original to us; indeed, in the final section of the ref. [2], those authors suggested that it would be interesting to explore these possibilities and, in a footnote, provided the formula for the one-loop correction. The idea is to have a CW-like model with gravity replacing electrodynamics in its effect on the scalar field. This idea was followed up in a number of papers [19, 38, 39]; however, there was never completed a fully self-consistent calculation that included quantum corrections to the background metric. Often feedback on the metric was assumed to be negligible.

Our approach is fundamentally different from previous treatments in several respects. We insist that the starting theory be classically scale invariant, so that this theory of gravity can be entertained as potentially complete without naturalness problems. The background curvature is to be determined self-consistently, ${ }^{15}$ and the Planck mass must be generated dynamically via DT. One reason for first discussing the gravitational theory without matter in the previous section was to gain some experience with the gravitational dynamics of such a model before embarking on other scenarios. So far, we have only treated maximally symmetric models, but, in principle, more complicated gravitational backgrounds can be considered. Our failure to find a locally stable vacuum state in the preceding section is consistent with the view that, without matter, gravity is bootless. Perhaps they can be tied together in a grand unified framework, but we feel there is much to be learned first in simpler models of this type before attempting that.

If DT does occur, then below the Planck scale, the theory will take the form of an effective field theory resembling the usual sort of scalar-tensor theory of gravity but with

\footnotetext{
${ }^{14}$ We note here that there has been recent work on inflation in the $R^{2}$ gravity context, in the light of the recent BICEP2 data [35, 36]. For other recent work on $R^{2}$ gravity and its supersymmetric extensions, see ref. [37].

${ }^{15} \mathrm{~A}$ similar scheme was attempted in Einstein gravity in [40].
} 
calculable corrections or matching conditions specified. We anticipate that the naturalness issues associated with physics below the Planck scale would return, so we cannot immediately suggest that this approach is a solution to the naturalness problems of particle physics. (Of course, as with supersymmetry breaking, one could arrange for this dynamics to be in a sector hidden from the Standard Model $[9,10]$.)

The action we shall consider is the sum of eq. (1.2) and eq. (2.3), $S=S_{\mathrm{ho}}+S_{m}$. This classical action has no masses and is formally invariant under global scale transformations, which we define as

$$
\phi(x) \rightarrow e^{\alpha} \phi(x), \quad g_{\mu \nu}(x) \rightarrow e^{-2 \alpha} g_{\mu \nu}(x),
$$

as reviewed in appendix $\mathrm{C}$.

The EoM associated with this action are

$$
\begin{aligned}
& -\left(\frac{2}{3 b} R-\frac{\xi \phi^{2}}{2}\right) R_{\mu \nu}+\frac{g_{\mu \nu}}{2}\left(\frac{1}{3 b} R-\frac{\xi \phi^{2}}{2}\right) R \\
& \quad+\frac{1}{a}\left[\frac{2}{3} R R_{\mu \nu}-2 R^{\kappa \lambda} R_{\mu \kappa \nu \lambda}+\frac{g_{\mu \nu}}{2}\left(R_{\kappa \lambda}^{2}-\frac{1}{3} R^{2}\right)\right]= \\
& =\frac{1}{2} T_{\mu \nu}-\left(\nabla_{\mu} \nabla_{\nu}-g_{\mu \nu} \square\right)\left(\frac{2}{3 b} R-\frac{\xi \phi^{2}}{2}\right)-\frac{1}{6 a}\left(2 \nabla_{\mu} \nabla_{\nu} R+g_{\mu \nu} \square R-6 \square R_{\mu \nu}\right), \\
& \text { where } T_{\mu \nu} \equiv \nabla_{\mu} \phi \nabla_{\nu} \phi-g_{\mu \nu}\left[\frac{1}{2}(\nabla \phi)^{2}+\frac{\lambda}{4} \phi^{4}\right],
\end{aligned}
$$

and

$$
-\xi \phi R-\square \phi+\lambda \phi^{3}=0
$$

If we take the trace of eqs. (5.2a), (5.2b) and combine the results, we find ${ }^{16}$

$$
-\xi \phi^{2} R+(\nabla \phi)^{2}+\lambda \phi^{4}=\square\left(\frac{4}{b} R-3 \xi \phi^{2}\right) .
$$

Writing $\square \phi^{2}=2 \phi \square \phi+2(\nabla \phi)^{2}$, we may rearrange the preceding equation as

$$
-\xi \phi^{2} R+6 \xi \phi \square \phi+\lambda \phi^{4}=\frac{4}{b} \square R-(1+6 \xi)(\nabla \phi)^{2} .
$$

Only for the conformal values, $(1 / b) \rightarrow 0,6 \xi=-1$, is eq. (5.4) equal to $\phi$ times the scalar EoM, eq. (5.2c), for arbitrary $\phi$. However, there are other solutions of these two equations that are mutually compatible. For example, if $\phi=\phi_{0}$, a constant value, then the gradients vanish, so eq. (5.2c) implies that $\xi \phi_{0} R_{0}=\lambda \phi_{0}^{3}$. Assuming that $\phi_{0} \neq 0$, the scalar curvature takes the constant value $R_{0}=\lambda \phi_{0}^{2} / \xi$. Then the trace equation eq. (5.4) is also satisfied. Returning to the tensor EoM, eq. (5.2a), for constant $\phi_{0}, R_{0}$, one can show after considerable algebra that this equation is also satisfied and yields no further information. In sum, constant $\phi_{0}$ with $R_{0}=\lambda \phi_{0}^{2} / \xi$ satisfies the classical EoM. This is a flat direction in the space of fields. As with the model without matter, the value of $R_{0}$ is classically undetermined, since the model remains scale invariant.

\footnotetext{
${ }^{16}$ The terms in $a$ drop out because of classical conformal invariance.
} 
Off-shell, for arbitrary constant $\phi$ and $R$, the matter action eq. (1.2) and its derivatives take the form

$$
\frac{S_{m}(r)}{V_{4}}=\frac{1}{R^{2}}\left[\frac{\lambda \phi^{4}}{4}-\frac{\xi \phi^{2}}{2} R\right]=\frac{1}{4}\left[\lambda r^{2}-2 \xi r\right], S_{m}^{\prime}(r)=\frac{1}{2}[\lambda r-\xi], S_{m}^{\prime \prime}(r)=\frac{\lambda}{2},
$$

where $r \equiv \phi^{2} / R$. This is the matter action and its derivatives for arbitrary ratio $r$, i.e., off-shell. It has an extremum for $r=\xi / \lambda$, which is a local minimum only if $\lambda>0$. (Subsequently, while searching for extrema of the effective action, we must keep in mind that $\lambda \geq 0$ for a classically stable ratio.) Adding the value of the gravitational action eq. (4.5), the total action takes the on-shell value

$$
S / V_{4}=\frac{1}{6}\left[\frac{2 w}{a}+\varepsilon-\frac{3 \xi^{2}}{2 \lambda}\right]
$$

Since the scale of the fields is undetermined at the tree level, the value of $\mu$ at which we are to evaluate the coupling constants is unknown. We can hope that both of these issues will be resolved by calculating the one-loop correction to the effective action and looking for a consistent DT solution. Since we now have dependence on $\{\lambda, \xi\}$ as well as the pure gravity couplings, the possibilities are much richer than in the model without matter.

Before embarking upon a fairly lengthy discussion and calculation, it may be useful to describe where we are headed. Assuming that the field $\phi \neq 0$, we can restore minimal coupling of the scalar field by transforming the matter action from the Jordan form, eq. (1.2), to the so-called Einstein frame by means of a conformal transformation $g_{\mu \nu}(x) \rightarrow \Omega^{-2} g_{\mu \nu}(x)$, where $\Omega^{2} \equiv \phi^{2} / M^{2}$, where $M$ is an arbitrary unit of mass introduced to keep the metric dimensionless. Then, after making this substitution, the classical matter action becomes

$$
S_{m}^{\mathcal{E}}=\int d^{4} x \sqrt{g}\left[\frac{1}{2}(\nabla \zeta)^{2}+\frac{\lambda M^{4}}{4}-\frac{\xi M^{2}}{2} R\right]
$$

where we defined $\zeta \equiv M \sqrt{6 \xi+1} \log (|\phi| / M)$. We recognize the linear term in $R$ as the Einstein-Hilbert action for gravity with $1 / G_{N}=8 \pi \xi M^{2} \equiv M_{P}^{2}$. Remarkably, the conformal transformation has transmogrified the self-interaction of the scalar into a cosmological constant. For $\lambda(\mu)>0$, such a model would naturally produce inflation. ${ }^{17}$ In units of the Planck mass $M_{P}$, the cosmological term is $\lambda M^{4} / 4=\left(\lambda / \xi^{2}\right)\left[M_{P}^{2} /(16 \pi)\right]^{2}$.

The original massless scalar has morphed into a massless dilaton $\zeta \propto \log |\phi|$, whose presence can be easily understood. The assumption that $\phi \neq 0$ corresponds to spontaneous breaking of scale invariance, and, since scaling is a valid symmetry classically, we must get a Goldstone boson in the broken phase. On the other hand, since the scale symmetry is explicitly broken by the anomaly in the QFT, we would expect the dilaton will actually have a nonzero mass that will be parametrically small compared to $M_{P}$. (In fact, this mass will be shown to arise at two loops.)

\footnotetext{
${ }^{17}$ As remarked below eq. (5.5), we must have $\lambda(v)>0$ for classical stability.
} 
As for the terms quadratic in curvature, in Einstein frame they become

$$
\begin{aligned}
S_{\mathrm{ho}}^{\mathcal{E}} & =\int d^{4} x \frac{\sqrt{g}}{a}\left[\frac{1}{2} C_{\kappa \lambda \mu \nu}^{2}+\frac{w}{3} \widetilde{R}^{2}+a \varepsilon \widetilde{G}\right] \\
\text { where } \quad \widetilde{R} & \equiv R-\frac{6}{M \sqrt{(1+6 \xi)}} \square \zeta+\frac{6}{M^{2}(1+6 \xi)}(\nabla \zeta)^{2}, \\
\widetilde{G} & \equiv G-8 \nabla_{\mu} J^{\mu},
\end{aligned}
$$

the term involving the Weyl tensor being invariant under conformal transformations. Assuming the conformal transformation does not change the Euler characteristic of the background topology, the change in $G$ must be of the form of a covariant derivative of a vector (see e.g., appendix B of [41]). ${ }^{18}$ At energies below the Planck scale, $\sqrt{\xi} M$, this takes the form of higher derivative terms in an effective field theory dominated by $S_{m}^{\mathcal{E}}$, eq. (5.7). For energies of order $M_{P}$, the situation becomes more subtle. As usual, in a flat background, it would appear as if there is a graviton plus massive scalar plus a massive spin-two ghost. However, because of the cosmological constant, $\lambda M^{4}$, Minkowski space is not a solution of the field equations, so the flat space interpretation may not be relevant. On the other hand, this depends on the size of the cosmological constant in units of the Planck mass, of order $\lambda / \xi^{2}$. If this ratio were small, as subsequent calculations suggest it might be, then it does seem as if there is a range of momenta, $\left(\lambda / \xi^{2}\right)^{1 / 4}<p / M_{P}<1$, where the background curvature might be negligible. However, the ghost mass is at the upper limit of the range of applicability of this analysis, so it is not so clear that the implied violation of unitarity is physically observable, even in principle. This regime is also subject to Hawking radiation from the horizon, which may cloud the issue further, although the temperature is relatively small. We are left uncertain but concerned about unitarity on the Planck scale. As a final comment concerning the Einstein frame, we note some recent work [42] concluding that (at least at one loop), results in the two frames (Jordan and Einstein) coincide on-shell.

To determine whether DT takes place, it is easiest to work with the action in Jordan form eq. (1.2), to which we return. As before, we write the effective action as

$$
\Gamma\left(\lambda_{i}, r, \rho / \mu\right)=S\left(\lambda_{i}, r\right)+B\left(\lambda_{i}, r\right) \log (\rho / \mu)+\frac{C\left(\lambda_{i}, r\right)}{2} \log ^{2}(\rho / \mu)+\ldots,
$$

where, again, $\rho=\sqrt{R}$. The collection of dimensionless coupling constants $\{a, w, \varepsilon, \xi, \lambda\}$ has been denoted by $\lambda_{i}$. With these conventions, the value of the effective action for $\rho=\mu$ is simply the classical action, $\Gamma\left(\lambda_{i}, r, 1\right)=S\left(\lambda_{i}, r\right) \equiv S_{m}\left(\lambda_{i}, r\right)+S_{\mathrm{ho}}\left(\lambda_{i}\right)$.

As mentioned earlier, although the RGE does provide an easy way to determine the coefficients $B\left(\lambda_{i}, r\right)$ and $C\left(\lambda_{i}, r\right)$, it is not really a substitute for the path integral calculation. In particular, the effective potential might have an imaginary part that cannot be removed by finite local counterterms but would not show up by this method. In fact, such contributions have plagued previous attempts to include matter [19], in particular, because of mixing with the conformal mode of the metric. In appendix $\mathrm{D}$, we have checked

\footnotetext{
${ }^{18}$ We found $J_{\mu}=\vartheta_{\nu} \nabla_{\mu} \vartheta^{\nu}-\vartheta_{\mu}(\nabla \cdot \vartheta)+\left(R_{\mu \nu}-g_{\mu \nu} R / 2\right) \vartheta^{\nu}+\vartheta_{\mu} \vartheta^{2}$, where $\vartheta_{\mu} \equiv \nabla_{\mu} \log (\Omega)$, differing slightly from the result of ref. [41]. The change $G \rightarrow \widetilde{G}$ plays no role in perturbation theory.
} 
by explicit calculation that there are no such modes arising in this model, so the following calculation does indeed give the correct result. However, we also learn that to avoid unstable modes (negative eigenvalues,) we must have all couplings $a, y, \xi, w$ positive and $0<w<3 / 2+3 \xi^{2} /(4 y)$, at least for some range of renormalization scales $\mu$. We again find five zero eigenvalues associated with $h_{1}$ in the conformal sector. Must these inequalities prevail at the DT scale? We shall return to this issue in section 7 .

It is slightly simpler algebraically to express the variations of the action in terms of $r$ and $\log \rho$ rather than in terms of $\phi$ and $R$. Since we seek solutions for which $(r, \rho)=$ $\left(r_{0}, v\right) \neq 0$, there is no loss of generality in so doing. The first derivatives are

$$
\begin{aligned}
& \frac{\partial}{\partial r} \Gamma\left(\lambda_{i}, r, \rho / \mu\right)=\frac{\partial}{\partial r} S_{m}\left(\lambda_{i}, r\right)+\log (\rho / \mu) \frac{\partial}{\partial r} B\left(\lambda_{i}, r\right)+\frac{\log ^{2}(\rho / \mu)}{2} \frac{\partial}{\partial r} C\left(\lambda_{i}, r\right)+\ldots, \\
& \rho \frac{\partial}{\partial \rho} \Gamma\left(\lambda_{i}, r, \rho / \mu\right)=B\left(\lambda_{i}, r\right)+C\left(\lambda_{i}, r\right) \log (\rho / \mu)+\ldots
\end{aligned}
$$

Note that $\partial S_{m}\left(\lambda_{i}, r\right) / \partial r$ is identical to $S_{m}^{\prime}(r)$ in eq. (5.5). Setting $(r, \rho)=\left(r_{0}, v\right)$ where these both vanish, and choosing the normalization scale $\mu=v$, we find

$$
\begin{aligned}
\left.\frac{\partial}{\partial r} \Gamma\left(\lambda_{i}, r, \rho / \mu\right)\right|_{r_{0}, v} & =\left.\frac{\partial}{\partial r} S_{m}\left(\lambda_{i}, r\right)\right|_{r_{0}, v}=0, \\
\left.\rho \frac{\partial}{\partial \rho} \Gamma\left(\lambda_{i}, r, \rho / \mu\right)\right|_{r_{0}, v} & =\left.B\left(\lambda_{i}, r\right)\right|_{r_{0}, v}=0 .
\end{aligned}
$$

These results are exact to all orders in the loop expansion. The form of these equations suggests a two-step approach to finding extrema: (1) Since $S_{m}\left(\lambda_{i}, r\right)$ is independent of $\rho$, the first equation eq. (5.11a) demonstrates that the value $r_{0}(\mu)=\phi^{2} / R=\xi(\mu) / \lambda(\mu)$ of the ratio at an extremum can be inferred in tree approximation, although we do not know the scale $\mu$ at which the couplings are to be evaluated. (2) The second equation eq. (5.11b) then determines the scale $\mu=v$ and the value of the curvature $\rho=v$, expressed as a special relationship among the couplings that must obtain at that scale.

In order to determine stability, we shall also need the matrix of second derivatives on-shell:

$$
\begin{aligned}
\left.\frac{\partial^{2}}{\partial r^{2}} \Gamma\left(\lambda_{i}, r, \rho / \mu\right)\right|_{r_{0}, v} & =\left.\frac{\partial^{2}}{\partial r^{2}} S_{m}\left(\lambda_{i}, r\right)\right|_{r_{0}, v}, \\
\left.\rho \frac{\partial^{2}}{\partial r \partial \rho} \Gamma\left(\lambda_{i}, r, \rho / \mu\right)\right|_{r_{0}, v} & =\left.\frac{\partial}{\partial r} B\left(\lambda_{i}, r\right)\right|_{r_{0}}, \\
\left.\rho^{2} \frac{\partial^{2}}{\partial \rho^{2}} \Gamma\left(\lambda_{i}, r, \rho / \mu\right)\right|_{r_{0}, v} & =C\left(\lambda_{i}, r_{0}\right) .
\end{aligned}
$$

Given our conventions, these equations eq. (5.12) are also exact to all orders in the loop expansion, but their leading nonzero contributions vary from tree level for those involving $S_{m}$, to one-loop for $B$, to two-loop ${ }^{19}$ for $C$. The second variation on-shell is therefore

$$
\delta^{(2)} \Gamma=\frac{1}{2}\left(\begin{array}{ll}
\frac{\delta \rho}{\rho} \delta r
\end{array}\right)\left[\begin{array}{cc}
C\left(\lambda_{i}, r_{0}\right) & B^{\prime}\left(\lambda_{i}, r_{0}\right) \\
B^{\prime}\left(\lambda_{i}, r_{0}\right) & S_{m}^{\prime \prime}\left(\lambda_{i}, r_{0}\right)
\end{array}\right]\left(\begin{array}{c}
\frac{\delta \rho}{\rho} \\
\delta r
\end{array}\right) .
$$

\footnotetext{
${ }^{19}$ As before, the two-loop contribution to $C_{2}$ can be calculated from one-loop corrections; $C_{3}$, from two-loop corrections, etc.
} 
This matrix has two eigenvalues $\varpi_{i}$ that may be approximated as

$$
\varpi_{1}\left(r_{0}, v\right)=\frac{S_{m}^{\prime \prime}}{2}+O\left(\hbar^{2}\right), \quad \varpi_{2}\left(r_{0}, v\right)=\frac{1}{2}\left[C_{2}-\frac{\left(B_{1}^{\prime}\right)^{2}}{S_{m}^{\prime \prime}}\right]+O\left(\hbar^{3}\right) .
$$

So $\varpi_{1}=\lambda(v) / 2$ is determined by the classical curvature, and $\varpi_{2}$, although of order $\hbar^{2}$, by one-loop results, just as with $C_{2}$.

To flesh this out, we need to determine $B$ and $C$ from the RGE: ${ }^{20}$

$$
-\left[\mu \frac{\partial}{\partial \mu}-\gamma_{\rho} \frac{\partial}{\partial \rho}\right] \Gamma\left(\lambda_{i}, r, \rho / \mu\right)=\left[\beta_{\lambda_{i}} \frac{\partial}{\partial \lambda_{i}}-\gamma_{r} r \frac{\partial}{\partial r}\right] \Gamma\left(\lambda_{i}, r, \rho / \mu\right) .
$$

As in the preceding section, the left-hand side may also be expressed as $\left(1+\gamma_{\rho}\right) \rho \partial \Gamma / \partial \rho$. The first variations eq. (5.11) vanish on-shell, so, to all orders,

$$
\left.\beta_{\lambda_{i}} \frac{\partial}{\partial \lambda_{i}} \Gamma\left(\lambda_{i}, r, \rho / \mu\right)\right|_{r_{0}, v}=0
$$

for arbitrary $\mu$. To one-loop order, eq. (5.15) becomes

$$
B_{1}+C_{2} \log (\rho / \mu)=\left[\beta_{\lambda_{i}}^{(1)} \frac{\partial}{\partial \lambda_{i}}-\gamma_{r}^{(1)} r \frac{\partial}{\partial r}\right]\left(S_{\mathrm{ho}}\left(\lambda_{i}\right)+S_{m}\left(\lambda_{i}, r\right)+B_{1} \log (\rho / \mu)\right)
$$

so that

$$
\begin{aligned}
& B_{1}\left(\lambda_{i}, r\right)=\beta_{\lambda_{i}}^{(1)} \frac{\partial}{\partial \lambda_{i}}\left[S_{\mathrm{ho}}\left(\lambda_{i}\right)+S_{m}\left(\lambda_{i}, r\right)\right]-\gamma_{r}^{(1)} r S_{m}^{\prime}\left(\lambda_{i}, r\right), \\
& B_{1}^{\prime}\left(\lambda_{i}, r\right)=\beta_{\lambda_{i}}^{(1)} \frac{\partial}{\partial \lambda_{i}} S_{m}^{\prime}\left(\lambda_{i}, r\right)-\gamma_{r}^{(1)} \frac{\partial}{\partial r}\left(r S_{m}^{\prime}\left(\lambda_{i}, r\right)\right), \\
& C_{2}\left(\lambda_{i}, r\right)=\left[\beta_{\lambda_{i}}^{(1)} \frac{\partial}{\partial \lambda_{i}}-\gamma_{r}^{(1)} r \frac{\partial}{\partial r}\right] B_{1}\left(\lambda_{i}, r\right) .
\end{aligned}
$$

As claimed, $C_{2}$ is determined by one-loop results. Taking note of eq. (5.11), these become on-shell

$$
\begin{aligned}
B_{1}\left(\lambda_{i}, r_{0}\right) & =\left.\beta_{\lambda_{i}}^{(1)} \frac{\partial}{\partial \lambda_{i}}\left[S_{\mathrm{ho}}\left(\lambda_{i}\right)+S_{m}\left(\lambda_{i}, r\right)\right]\right|_{r_{0}, v}, \\
B_{1}^{\prime}\left(\lambda_{i}, r_{0}\right) & =\left.\beta_{\lambda_{i}}^{(1)} \frac{\partial}{\partial \lambda_{i}} S_{m}^{\prime}\left(\lambda_{i}, r\right)\right|_{r_{0}, v}-\gamma_{r}^{(1)} r_{0} S_{m}^{\prime \prime}\left(\lambda_{i}, r_{0}\right), \\
C_{2}\left(\lambda_{i}, r_{0}\right) & =\left.\beta_{\lambda_{i}}^{(1)} \frac{\partial}{\partial \lambda_{i}} B_{1}\left(\lambda_{i}, r\right)\right|_{r_{0}, v}-\gamma_{r}^{(1)} r_{0} B_{1}^{\prime}\left(\lambda_{i}, r_{0}\right) .
\end{aligned}
$$

Thus, on-shell, $B_{1}$ is independent of $\gamma_{r}$, but $C_{2}$ is not. This reflects the fact that, from eq. (5.11b), the condition $B_{1}=0$ is one of the (leading order) conditions for an extremum. From eq. (5.14), on the other hand, we see that (unlike in the pure gravity case discussed in the last section) the sign of $C_{2}$ does not determine the nature of the extremum; we must calculate $\varpi_{2}$. We find

$$
\varpi_{2}=\left.\frac{1}{2}\left[\left(\beta_{\lambda_{i}}^{(1)} \frac{\partial}{\partial \lambda_{i}}\right)^{2}\left[S_{\mathrm{ho}}\left(\lambda_{i}\right)+S_{m}\left(\lambda_{i}, r\right)\right]-\frac{1}{S_{m}^{\prime \prime}}\left(\beta_{\lambda_{i}}^{(1)} \frac{\partial}{\partial \lambda_{i}} S_{m}^{\prime}\left(\lambda_{i}, r\right)\right)^{2}\right]\right|_{r_{0}, v} .
$$

\footnotetext{
${ }^{20}$ As before, we shall suppress possible gauge parameters. The only gauge-dependent quantities here are the wave function renormalizations, which we shall show do not contribute to observables.
} 
The order of operations is important; the derivatives with respect to the couplings must be carried out before setting $r=r_{0}$. Note that the $\gamma_{r}$-dependence has cancelled out between the two terms in eq. (5.14), as we anticipated, because the result eq. (5.20) must be gauge invariant.

There is still quite a lot of work to be done to evaluate and solve eqs. (5.11a), (5.11b) for potential extrema and to evaluate eq. (5.20) to determine local stability. First of all, we need the one-loop beta-functions. These have been given several places in the literature and, for easy reference, are reviewed in appendix $\mathrm{E}$ in the present notation. Quite generally, we see that $\beta_{a}$ and $\beta_{\varepsilon}$ have the same form as in eq. (4.18a), but with the positive constants $\beta_{2}$ and $\beta_{1}$ dependent upon the matter content. In this model with one real scalar only, $\beta_{2}=799 / 60$ and $\beta_{1}=523 / 120$. Thus, the coupling $a$ is always AF and, at one-loop, $\beta_{a}$ is independent of the other coupling constants, a residue of the conformal invariance of the Weyl tensor. Noting that $a_{0} / a=1+a_{0} \beta_{2} t$, where $d t=\kappa d(\ln \mu)$, it proves useful to define a new parameter

$$
u \equiv\left(1 / \beta_{2}\right) \log \left(a_{0} / a\right)=\left(1 / \beta_{2}\right) \log \left(1+a_{0} \beta_{2} t\right)
$$

so that $d u=a d t=-d a /\left(\beta_{2} a\right)$. The coupled equations simplify considerable if we rescale $\lambda$ as we did with $b, y \equiv \lambda / a$. Then the three remaining variables $w, \xi, y$ obey

$$
\begin{aligned}
\frac{d w}{d u} & \equiv \bar{\beta}_{w}=\frac{10}{3}\left[w^{2}-\frac{1099}{200} w+\frac{1}{8}+\frac{1}{5}\left(\frac{6 \xi+1}{4}\right)^{2}\right] \\
\frac{d \xi}{d u} & \equiv \bar{\beta}_{\xi}=\left[(6 \xi+1) y-\xi\left(\frac{3 \xi^{2}}{2}+4 \xi-3+\frac{10 w}{3}-\frac{1}{4 w}\left(9 \xi^{2}+20 \xi-4\right)\right)\right] \\
\frac{d y}{d u} & \equiv \bar{\beta}_{y}=\left[18 y^{2}+y\left(\frac{499}{60}-3 \xi^{2}+\frac{1}{2 w}\left(1+12 \xi+33 \xi^{2}\right)\right)+\frac{\xi^{2}}{2}\left(5+\frac{(6 \xi+1)^{2}}{4 w^{2}}\right)\right] .
\end{aligned}
$$

Note that a no longer appears in these "reduced" beta-functions. This suggests that there may well be fixed points at finite $w, \xi, y$, where all three beta-functions simultaneously vanish.

Although our primary interest is in finding where $B_{1}\left(\lambda_{i}, r_{0}\right)=0$, let us first explore whether there are fixed points. First, note that, if $\xi=0$ (minimal coupling), then $\bar{\beta}_{\xi}=0$ implies $y=0$ as well. $\bar{\beta}_{y}$ also vanishes for $\xi=y=0$. Then $\bar{\beta}_{w}=0$ implies $w \approx 0.02514$ or $w \approx 5.46986$. Other fixed points are more difficult to locate and must be found numerically, but we found four more. All the fixed points are shown in table 1.

Of the six, it can be shown that all are saddle points except for the one located at $w \approx 0.0245, \xi \approx-0.0252, y \approx-1.273$, which is UV attractive. Unfortunately, since $y<0$ near there, it has the opposite sign to the one required for stability in $r$, eq. (5.5). So this does not appear to be an acceptable model for large scales. Further, as we shall explain in section 7 , no renormalization trajectory can cross from $y>0$ to $y<0$. (This would require a change in sign of the curvature, so it is not surprising that it is different phase.)

Returning to the determination of $B_{1}$, from eq. (5.5), we have the values of the matter action and its derivatives and, as noted previously, $r_{0}=\xi / \lambda$, which implies $a(\mu) r=$ 


\begin{tabular}{|l|c|c|c|c|}
\hline & $w$ & $\xi$ & $y$ & $z$ \\
\hline 1. & 0.02514 & 0. & 0. & n.a. \\
\hline 2. & 0.36011 & 1.7907 & -4.8714 & -1.3710 \\
\hline $\mathbf{3 .}$ & $\mathbf{0 . 0 2 4 5 0}$ & $-\mathbf{0 . 0 2 5 1 9}$ & $\mathbf{- 1 . 2 7 2 6}$ & $-\mathbf{0 . 0 1 5 2 6 5}$ \\
\hline 4. & 0.03336 & 0.1898 & -0.2643 & -3.0652 \\
\hline 5. & 5.4699 & 0. & 0. & n.a. \\
\hline 6. & 5.4705 & -0.02567 & -0.4654 & $-1.941 \times 10^{-4}$ \\
\hline
\end{tabular}

Table 1. Fixed points.

$\xi(u) / y(u)$. From eq. (5.19a), we may write the on-shell value

$$
\begin{aligned}
& B_{1}\left(\lambda_{i}, r_{0}\right)=\beta_{a} \frac{\partial}{\partial a}\left[S_{\mathrm{ho}}+S_{m}\right]+\beta_{w} \frac{\partial}{\partial w} S_{\mathrm{ho}}+\left[\beta_{\xi} \frac{\partial}{\partial \xi}+\beta_{y} \frac{\partial}{\partial y}\right] S_{m}, \\
& \text { or } \quad \frac{B_{1}}{\kappa V_{4}}=\frac{\beta_{2}}{6}\left[2 w-\frac{\beta_{1}}{\beta_{2}}-\frac{3 \xi^{2}}{2 y}\right]+\frac{1}{3} \bar{\beta}_{w}+\frac{\xi}{4 y}\left(\frac{\xi}{y} \bar{\beta}_{y}-2 \bar{\beta}_{\xi}\right),
\end{aligned}
$$

where the $\bar{\beta}_{i}$ are given in eq. (5.22). From their form, one can observe that $B_{1}=B_{1}(w, \xi, y)$ has no explicit dependence on $a$. The equation to be solved, $B_{1}=0$, is of the form $P(w, \xi, y) / w^{2} y^{2}$, where $P$ is a polynomial in the three variables with highest degree $w^{4} \xi^{4} y^{2}$. Not surprisingly, there is a continuum of (real) solutions satisfying $P(w, \xi, y)=0$. Because the classical action on-shell depends on $\xi, y$ only through the ratio $\xi^{2} / y$, this space of solutions is more easily represented in terms of different variables. Changing from $y$ to $z$ with $z \equiv 3 \xi^{2} /(4 w y)$, we find that

$$
\frac{B_{1}}{\kappa V_{4}}=\left(\frac{20 w^{2}+(1+6 \xi)^{2}}{18}\right)(z+1)^{2}+\frac{z}{3}\left(8 \xi(w-1)-11 w+\frac{13}{6}\right)-\xi-\frac{5 w}{3}-\frac{151}{240},
$$

which is only quadratic in each parameter and non-degenerate in $z$, since, as discussed earlier, $w \neq 0$ in perturbation theory. The contour plot of $B_{1}=0$ is a very large region, much of which is not of particular physical interest. As remarked below eq. (5.5), we may restrict our search to $\lambda>0$, i.e., $y>0$. Therefore, the signs of $z$ and $w$ must agree. Further, in order to recover Einstein gravity below the DT scale, we must have $\xi>0$. Although we allow $w$ to have either sign, it is most convenient to display the contour plot of perturbative solutions for the regions $w>0$ and $w<0$ separately. For $w>0$, we find the contour plot of solutions in figure 1 . It is noteworthy that the entire space of solutions lies within the limits $0<w \lesssim 1.79,0<\xi \lesssim 0.862$, and $0<z \lesssim 2.82$.

For $w<0$, the contour plot is more complicated, especially because the equation $B_{1}=0$ degenerates at $z=-1$, where it becomes simply $6 w+5 \xi-8 w \xi=973 / 240$. For all $w<0$, this has solutions $\xi=(1 / 240)(973-1440 w) /(5-8 w)$, so that $\xi$ lies within the fairly narrow range $3 / 4<\xi<0.811$. A portion of the general contour plot for $w, z<0, \xi>0$ is shown in figure 2 .

The next step is to determine the subspace of the preceding solutions to $B_{1}=0$ that are local minima, viz., those having $\varpi_{2}>0$, in eq. (5.20). The explicit expression is 


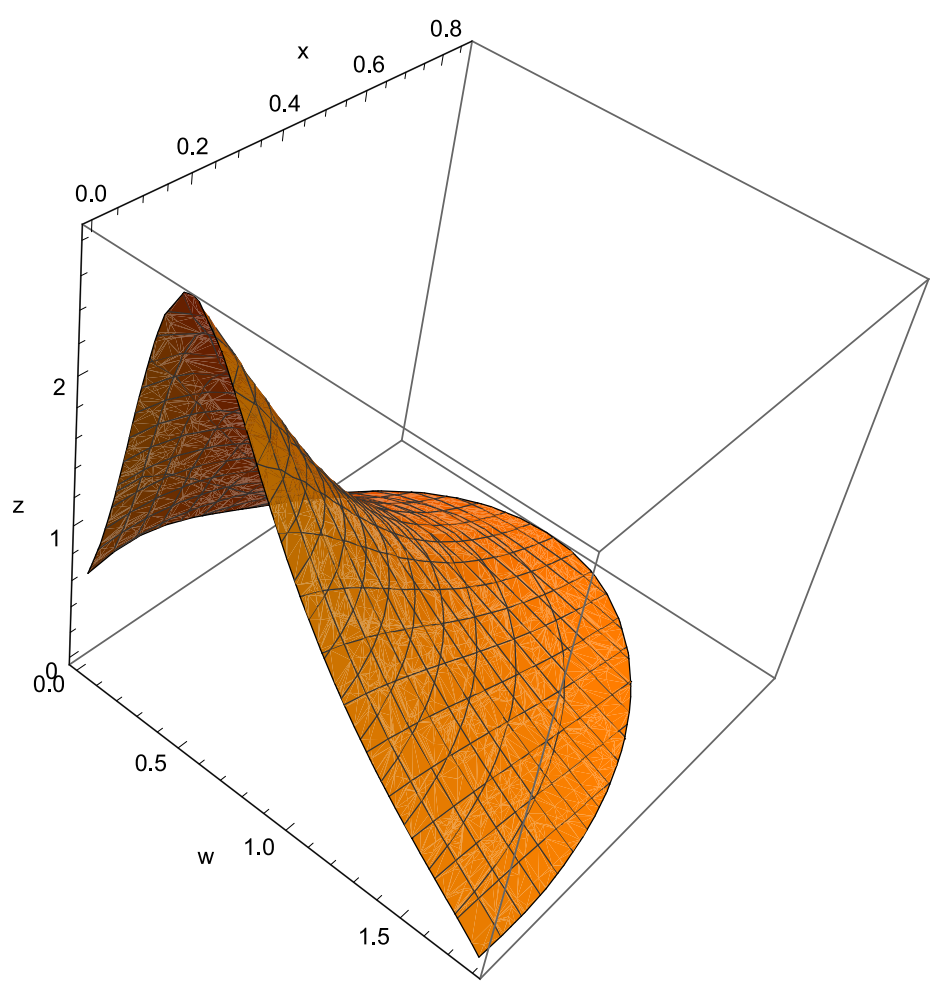

Figure 1. $B_{1}=0$ for $w>0$.

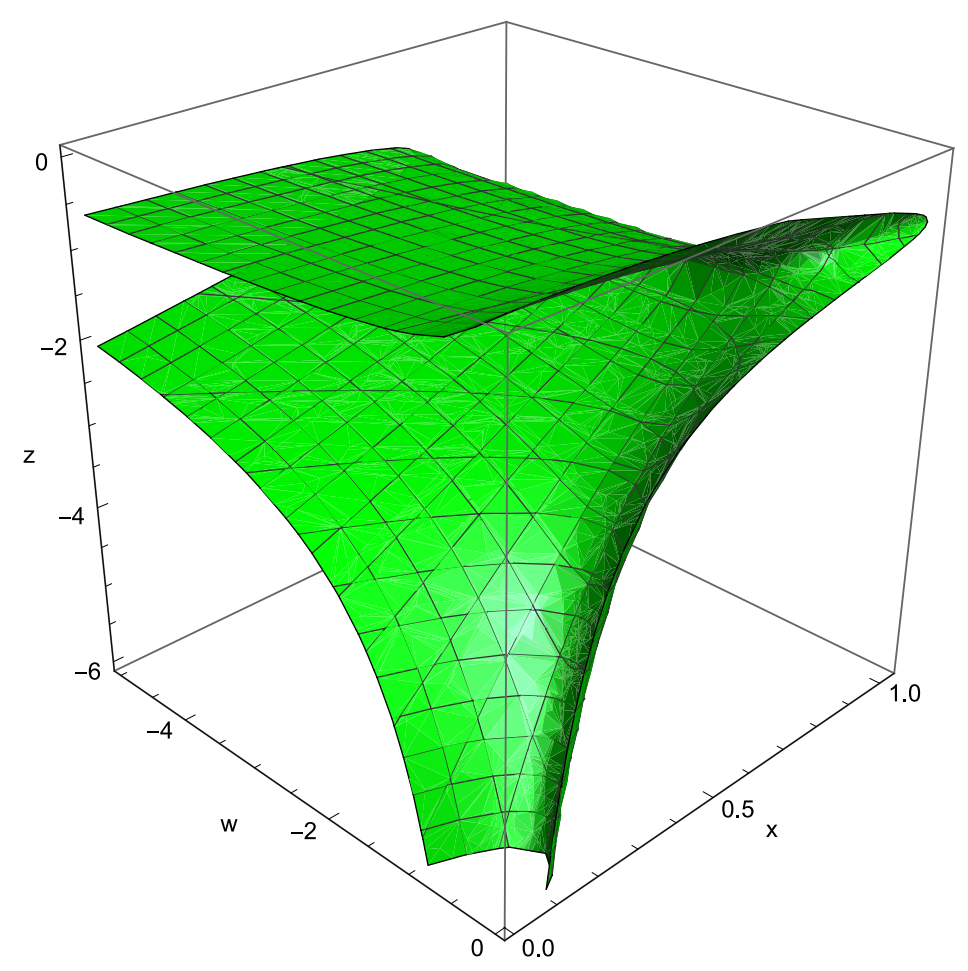

Figure 2. $B_{1}=0$ for $w<0$. 
straightforward to calculate but messy:

$$
\begin{aligned}
\varpi_{2}(w, \xi, z)=\frac{\kappa^{2} a}{4320 w z}[ & 540 \xi^{2}\left(-1+6 \xi+72 \xi^{2}\right) \\
+10 z\left(1600 w^{4}-9992 w^{3}+2 w^{2}\left(3407+600 \xi-2160 \xi^{2}\right)\right. & \\
& +18 \xi\left(19-149 \xi+375 \xi^{2}+324 \xi^{3}\right) \\
& \left.-3 w\left(55+528 \xi-2400 \xi^{2}+1116 \xi^{3}+432 \xi^{4}\right)\right) \\
-z^{2}\left(16000 w^{4}+160 w^{3}(289+600 \xi)\right. & \\
& +4 w^{2}\left(2363-33624 \xi+71280 \xi^{2}+2160 \xi^{3}\right) \\
& +1080 w\left(-45+112 \xi-344 \xi^{2}+176 \xi^{3}+24 \xi^{4}\right) \\
& \left.+15\left(355-1204 \xi+5820 \xi^{2}-10800 \xi^{3}+5184 \xi^{4}\right)\right) \\
-10 z^{3}\left(101+36 \xi\left(34+74 \xi-105 \xi^{2}+414 \xi^{3}\right)\right. & \\
& +4\left(1200 w^{4}+2 w^{3}(-191+720 \xi)+10 w^{2}\left(53+36 \xi+576 \xi^{2}\right)\right. \\
& \left.\left.+3 w(1+6 \xi)\left(-43-270 \xi+192 \xi^{2}+18 \xi^{3}\right)\right)\right) \\
-40 z^{4}( & \left.\left.20 w^{2}+(1+6 \xi)^{2}\right)^{2}\right] .
\end{aligned}
$$

Since we require $y>0$, it follows that $w z>0$, so the polynomial ${ }^{21}$ in brackets must be positive for the extremum to be a local minimum. The intersection of the region $\varpi_{2}(w, \xi, z)>0$ with the $B_{1}(w, \xi, z)=0$ surface is shown in figure 3 for $w>0$ and in figure 4 for $w<0$.

Therefore, we have shown that there remains a continuum of local minima at which DT takes place. All such points are candidates for no-particle solutions (vacua) in this model. To illustrate, some values for $w>0$, for $z \approx .0005$, one has $B_{1}=0$ and $\varpi_{2}>0$ for $0<w<1.78$ with $\xi=0.0834-0.000333 w+\sqrt{0.293+0.833 w-0.556 w^{2}}>0$. At the other extreme, for $z \approx 2.82$, one finds $0.357<w<0.364$, with $\xi=-0.0207-0.129 w+$ $\sqrt{-0.0756+0.417 w-0.539 w^{2}}>0$.

As a renormalizable completion of Einstein gravity, this model is unsatisfactory for a reason that is not immediately apparent. The only UV fixed point has $y<0$, whereas all local minima must have $y>0$. One can show that as the couplings evolve from lower to higher scales, no path runs from $y>0$ to $y<0$. Therefore, the region of parameter space in which DT occurs is not connected to the region in which AF holds. Invariably, one or another of the couplings grows and perturbation theory breaks down. (We have not investigated whether calculable nonperturbative effects, such as instantons, might alter this conclusion, but it seems doubtful.) This property does not appear to be a generic property of any such model, and we can hope (along with previous authors [3]) that a richer theory of matter, such as a grand unified theory, might avoid such a conclusion.

\footnotetext{
${ }^{21}$ It is of fourth-degree in each of the three parameters $w, \xi, z$ except at $z=-1$, where it becomes cubic in $w, \xi$.
} 


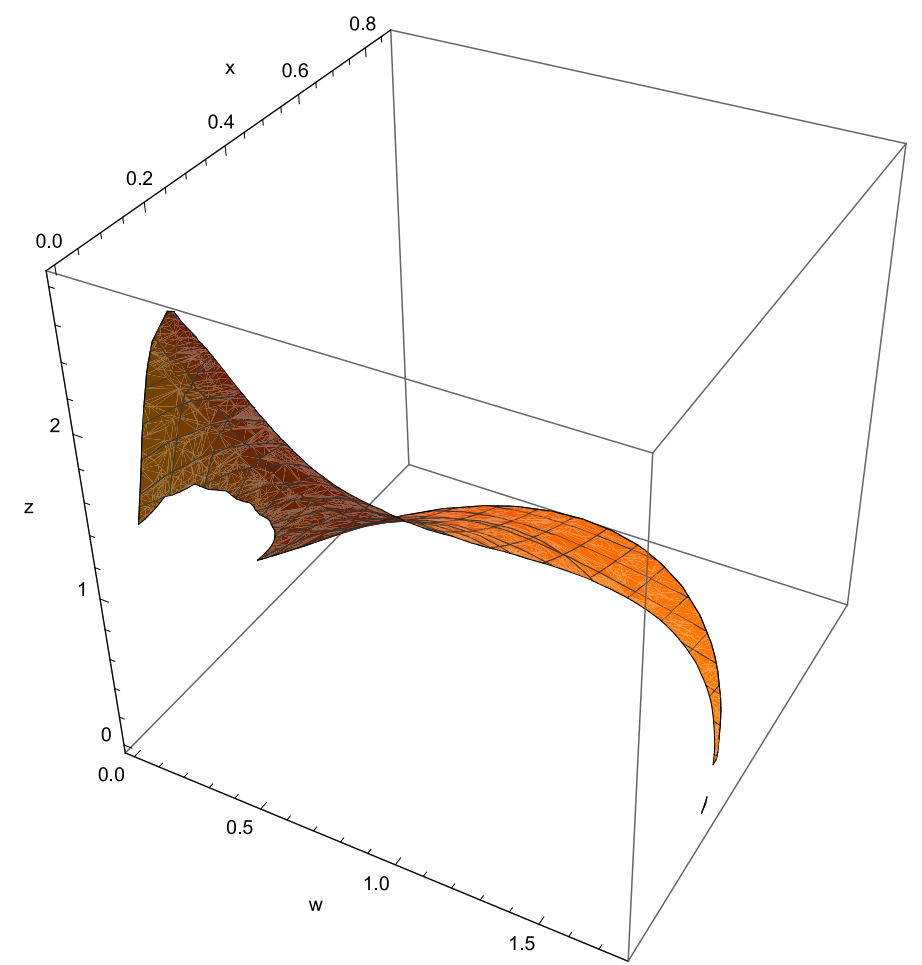

Figure 3. $B_{1}=0$ for $w>0, \varpi_{2}>0$.

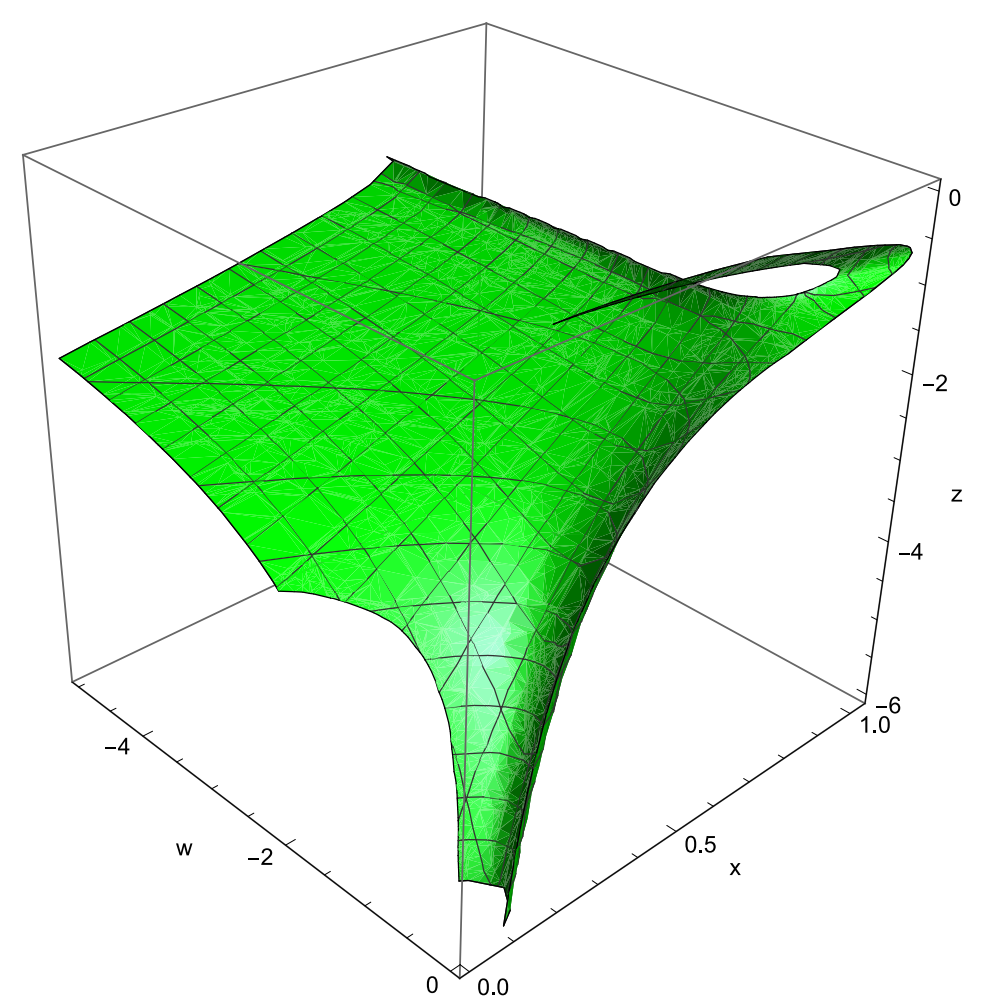

Figure 4. $B_{1}=0$ for $w<0, \varpi_{2}>0$. 


\begin{tabular}{|l|c|c|c|c|}
\hline & $w$ & $\xi$ & $y$ & $z$ \\
\hline 1. & 0.0271 & 0. & 0. & n.a. \\
\hline 2. & 0.7497 & 1.2518 & -0.7150 & -2.1926 \\
\hline $\mathbf{3 .}$ & $\mathbf{0 . 0 2 4 2}$ & $\mathbf{- 0 . 0 2 8 5}$ & $\mathbf{- 1 . 5 1 2 9}$ & $-\mathbf{0 . 0 1 6 6}$ \\
\hline 4. & 0.0567 & 0.1771 & -0.1103 & -3.764 \\
\hline 5. & 6.9280 & 0. & 0. & $n . a$. \\
\hline 6. & 6.9309 & -0.0299 & -0.7348 & $-1.318 \times 10^{-4}$ \\
\hline
\end{tabular}

Table 2. Fixed Points with Standard Model matter

\section{Additional matter}

It is straightforward to generalize the model above to a more general theory containing gauge, Yukawa and additional scalar multiplets if we assume that the scalar sector we have described is a hidden sector, interacting with what we may call the matter sector only via gravitational interactions. This is because at one-loop order, the $\beta$-function and effective potential calculations we have described are unaffected, except for matter contributions to the $\beta_{a, b, \varepsilon}$. (We assume here that the dominant non-minimal $\xi$-type coupling at the DT scale is to the original $\phi$-singlet.) We reproduce these generalized $\beta$-functions in appendix E. Generally speaking the results remain qualitatively the same. For example, with $N_{0}=$ $5, N_{1 / 2}=24, N_{1}=0, N_{1}^{0}=12$, corresponding to a coupling of our theory to the Standard Model (including right-handed neutrinos), we find

The fixed point with (now) $w \approx 0.0242$ remains UV attractive, although two of the eigenvalues of its stability matrix develop imaginary parts. This simply means that the couplings oscillate around an envelope that is AF.

As in our original model, there is a substantial range of parameter space such that $B_{1}=0$ represents a perturbatively stable minimum of the effective potential. Thus it is feasible to entertain the possibility that a realistic theory might be constructed with a "hidden sector" responsible for generating the Planck mass via DT.

Of course if we wished to take seriously the above possibility in the SM context, we would need to consider the indication of new physics associated with the electroweak vacuum stability issue, caused by the running to negative values of the Higgs quartic coupling $\lambda_{H}(\mu)$. A recent comprehensive analysis [43] suggests the possibility that a new physics threshold is required at a scale of around $\Lambda_{I} \approx 10^{10}-10^{12} \mathrm{GeV} .{ }^{22}$ Now the DT scale in our model is given by

$$
\Lambda_{\mathrm{DT}} \sim \sqrt{R} \sim \sqrt{\frac{\lambda}{\xi}}\langle\phi\rangle \sim \sqrt{\lambda} M_{P} / \xi,
$$

so to make this scale coincide with $\Lambda_{I} \sim 10^{12} \mathrm{GeV}$ requires $\sqrt{\lambda} / \xi \sim 10^{-7}-10^{-9}$. Proponents of Higgs inflation [46-50] are content to contemplate large values of $\xi\left(\xi \approx 10^{4}\right)$, but it is

\footnotetext{
${ }^{22}$ Note that while $\lambda_{H}(\mu)$ is of course gauge invariant for all $\mu$, defining the instability scale by, for example, $V\left(\Lambda_{I}\right)=0$ is manifestly gauge dependent, so care is required [44, 45].
} 
clear from, for example eq. (5.22), that such $\xi$ values lead to loss of perturbative credibility for our calculations.

Evidently it will also be interesting to entertain a more complicated generalization where the non-minimally coupled scalar sector has gauge and Yukawa interactions. For example, one could imagine a Grand Unified Theory (GUT) wherein the DT-generated vev for the scalar fields both generated Einstein gravity and broke the GUT gauge invariance down to the SM. We postpone this possibility for future discussion.

\section{Constraints on coupling constants}

What constraints exist on the couplings constants? First of all, unlike flat space field theories, not all spacetimes can be analytically continued from Lorentzian to Euclidean signature. We tacitly assume that all physically realizable spacetimes arise by the reverse process of continuation from a Euclidean metric. We are especially interested in models in which the couplings are asymptotically free so that perturbation theory can be used to determine the solutions. We have already discussed some properties of the effective action at the DT scale in two cases, the pure $R^{2}$-model of gravity and the $R^{2}$ plus a real scalar. We also touched on inclusion of the SM fields in a hidden sector.

In the case of no matter, we found that there were no local minima of the effective action, regardless of the signs of the couplings.

In the case of the real field and its simple extension discussed in section 6 , the basin of attraction of the AF fixed point is a distinct phase from the range of parameters where DT occurs. This can be seen as follows: we required $y>0$ at the DT scale for stability. If the couplings are to approach the AF fixed point where $y<0$, then the trajectory as some point will have to cross $y=0$. If at some point $y \rightarrow 0$ for positive $y$, then we see from eq. (5.22c) that $\bar{\beta}_{y}>0$ for all values of $\xi, w$, so $y$ must increase from such a point. Therefore, $y$ cannot become negative, at least, not so long as perturbation theory is valid.

There has been considerable discussion in the literature ${ }^{23}$ of whether AF for all couplings obtains, but we have not seen previous discussions of whether or not the couplings actually run from their on-shell values to their AF values. Our result appears to be modeldependent, and there can be hope that this obstruction will be remedied in future, more realistic models. Nevertheless, this is an issue that requires attention, even in the existing models.

We found in appendix $\mathrm{D}$ that the EPI for $\Delta \Gamma$ was convergent only if all couplings $a, w, \xi, y$ are positive with $w<3 / 2+3 \xi^{2} /(4 y)$. We postponed the question of whether this is required to be true at all scales or, in particular, at the DT scale $\mu=v$. We believe the answer in both cases is "no", based on experience with flat space models. First, consider the familiar double-well potential $V=\lambda \phi^{4} / 4-m^{2} \phi^{2} / 2$ with $\lambda, m^{2}>0$, we know that it is stable near the classical minima $\pm v$. Between the two minima, the true effective potential is simply a straight line between the two minima, but the perturbative effective potential

\footnotetext{
${ }^{23}$ See Chapter 9 of ref. [6] for a summary of some models.
} 
resembles the classical potential. The one-loop correction to the effective potential is

$$
\Delta V^{(1)}=\frac{\left(3 \lambda \phi^{2}-m^{2}\right)^{2}}{64 \pi^{2}} \log \left(3 \lambda \phi^{2}-m^{2}\right)
$$

This does have physical meaning in certain situations [51], even when $\Delta V^{(1)}$ becomes imaginary. The imaginary part represents half the decay rate per unit volume, as expected, although the decay process is rather complicated. Nevertheless, unstable modes do not necessarily invalidate the perturbative result when properly interpreted.

Second, consider the case of DT in massless scalar electrodynamics [13], which is a model that is not AF. In general, we believe that the self-coupling of the scalar field $\lambda(\mu)$ must be positive for the convergence of the EPI and for the potential to be bounded below. However, if one adopts a renormalization scheme similar to the one used here, the selfcoupling $\lambda(\mu)$ turns negative at the DT scale [52]. This is permissible because $\lambda(v)$ is unusually small at the minimum $v$, comparable in size with the electromagnetic one-loop correction; $\lambda(v) \sim-\kappa \alpha^{2}(v)$. At somewhat lower scales, $\lambda(\mu)$ is positive, typically on order of $\alpha$, and larger than the one-loop correction. However, at very small or very large scales, it becomes large, and perturbation theory breaks down.

With these cautionary examples in mind, let us consider the immediate applications in this paper. There are many well-known problems [14] defining functional integrals, especially when gravity is included. Among them is that the manifold over which one integrates and the determination of the metric are intertwined, so we seem to be caught in a vicious circle. Further, in our case, the action eq. (2.3) includes the "topological term" $G$ and possibly also boundary or surface integrals. Beyond perturbation theory, we have little to add to these issues. Within perturbation theory, it seems that the effects of the topological term $G$ can be restricted to the "classical" background, and we do not need to address the topology of the quantum fields. In the background field method, summarized in appendix B, it is required to evaluate the auxiliary functional eq. (B.2). This will converge if the source-free EPI $\Delta \mathcal{G}\left[\phi_{i}, 0\right]$ converges, since $\Delta S$ is at least quadratic in the quantum fields. ${ }^{24}$

For the matter-free case in section 2, it is necessary to find a scale where $a>0$ and $0<w(\mu)<3 / 2$ in order to evaluate the integral, and that is possible. Now if $a>0$ at one scale, its sign cannot change (so long as perturbation theory holds.) For $0<w<3 / 2$, the EPI must agree with our determination of $B_{1}$ and $C_{2}$ in eqs. (4.19), (4.20) via the RGE. For $w(\mu)$ outside this range of values, the effective action $\Gamma$ will develop an imaginary part, as evidenced by unstable modes in the EPI. Solving the equation $B_{1}=0$, we found extrema at $w_{-} \approx-0.3$ and $w_{+} \approx 1.8$, both outside the range of convergence of the EPI. Accordingly, our RGE calculation gives only the real part of $\Delta \Gamma$ and does not tell us that there is an imaginary part as well. However, we did determine that both extrema were local maxima, $C_{2}<0$, so we should expect $\Delta \Gamma$ to have an imaginary part. It is not given simply by replacing $\rho$ by $-\rho$ in the logarithms in eq. (4.6). In terms of the eigenmodes of the Laplacian outlined in appendix $\mathrm{D}$, the coefficient of the imaginary part will come from only

\footnotetext{
${ }^{24}$ Fermion fields can be included without changing the basic results, but they would require a separate discussion.
} 
those modes that are negative. Since all modes for sufficiently large $n$ are positive, there are only a finite number of unstable modes, so that this is consistent with renormalizability.

For the real field in section 5 , we required $y>0$ for stability of the ratio $r=\phi^{2} / R$. The constraints on the tensor sector for convergence of the EPI are $a>0, w<3 / 2+3 \xi^{2} /(4 y)$. (See eq. (D.4).) From the conformal sector, eq. (D.5), we must have $w>0$ and $\xi>0$. We also learn that there are six zero modes, one, the dilaton, associated with SSB of the classical scale invariance, and the other five as in the matter-free case, associated with $\varphi_{1}$. As explained in appendix D, the dilaton will get a mass ${ }^{2}$ at two-loop order proportional to $C_{2}$. We do not know what will happen to the other five modes, whose origin remains obscure to us.

Although we found an AF fixed point, it has $y<0$ and $\xi<0$, outside the bounds above. Thus, independently of the existence of DT, the EPI does not converge for values of the couplings near the AF fixed point! That is decidedly unsatisfactory for a perturbative solution to exist.

Are these inequalities also necessary at the DT scale? We did find once again that $a>0$ and $y>0$. We also required $\xi>0$, so that the gravitational constant has the correct sign. On the other hand, it is not clear that we must have $w(v)>0$. Within these restrictions, we found a large region of parameter space where DT can occur $\left(B_{1}=0\right)$ and where the points are local minima. Thus, this is a viable mechanism for generation of the Planck scale. Which of these candidate vacua might be acceptable would require a cosmological analysis, but this is not a realistic model anyway.

In summary, the question of constraints on the couplings is thus scale dependent and depends on the phenomena of interest. However, to calculate radiative corrections, one may make the separation between the classical background and quantum corrections at any convenient scale and later determine whether the questions of interest are at scales at which the couplings are still small. Especially for AF theories, starting at a very large scale where the couplings are small is an attractive possibility, but, unlike the models in this paper, we would want the EPI over the quantum field converge in that domain.

Unfortunately, in the examples studied in this paper, one or another physical or aesthetic requirement was violated. For the pure metric model, section 2, the extrema were maxima rather than minima. With the addition of a real scalar, we found that we were able to find a purely real radiative correction $\Delta \Gamma$ for values of the couplings where there were local minima of the effective action, but this region turned out not to be continuously connected to the AF domain, so that, above the Planck scale, these theories appear to become strongly coupled. Whether they must be regarded as incomplete, we cannot say, but this result is disappointing. We do not know whether the problem lies with our insistence on maximal isometry for the background, but it would be rather surprising if the most symmetric situation has problems not shared by less symmetric backgrounds.

This does not invalidate our conclusion that DT can occur perturbatively. At the DTscale $v$, one-loop corrections are crucial, and the couplings need not respect the inequalities required near the AF fixed point. The only inequalities we can impose at the DT scale are those required for stability at that scale, such as $y(v)>0, \varpi_{2}(v)>0$. 


\section{Conclusions}

In this paper, we have presented a number of new formal results for classically scaleinvariant, renormalizable gravity models. Such models can be motivated by the fact that the scale breaking (due to the anomaly of the corresponding QFT) is soft, preserving naturalness, unlike models that include explicit scale-breaking in the action. They are also attractive, in that $R^{2}$-gravity is not only renormalizable but also asymptotically free $(\mathrm{AF})$, a quality generally preserved when renormalizable interactions involving matter fields are added.

We extended the formalism for determining whether dimensional transmutation (DT) takes place to include the background metric itself, at least for maximally symmetric backgrounds. We analyzed the situation in the absence of matter, showing that there was not a perturbative background that was locally stable.

Classically, a scale-invariant theory that is spontaneously broken yields a massless Goldstone boson. Since DT is a form of spontaneous symmetry breaking, there remains such a massless particle in lowest order in perturbation theory in the QFT. However, since the QFT breaks scale invariance due to the anomaly, this particle becomes massive from radiative corrections (that are second-order in the loop-expansion.) We showed how this mass-squared could be determined from a certain collection of the one-loop results, without having to face the daunting task of computing the full two-loop corrections to the effective action. This allows one to calculate the local curvature at the DT scale, which determines whether or not an extremum corresponds to a local minimum or maximum of the effective action In a kind of corollary to the discussion here, we elaborate in a companion paper [22] how the effective action of this theory may be regarded in a sense as involving only two gravitational couplings rather than three, and how this observation relates to a possible $a$-theorem for $R^{2}$ gravity.

The preceding observations and formulas can be extended to $R^{2}$-models that include matter, as was illustrated by considering the simplest case of the addition of a real scalar field. Despite its simplicity, several properties not previously explored concerning $R^{2}$ models emerged. The non-minimal coupling $\xi$, and the ratios of couplings, $w=a / b$ and $y=\lambda / a$, have a number of finite fixed points, only one of which is UV attractive. The basin of attraction of this AF fixed point is limited, and, in fact, does not include the region in which DT minima occur. Accordingly, the couplings in the regions where DT occurs are not AF and become either strongly coupled or somehow modified at high scales. We did not attempt to determine the behavior at strong coupling; it may depend on which couplings become large at high scales. Treating this as an effective field theory, we then showed that DT can occur over a very wide range of parameters and that a large subset of these extrema are in fact minima of the effective action.

In future work, we shall examine theories including other matter fields. Some models that included non-Abelian gauge fields were partially investigated in refs. [6, 38, 39]. Some of these are claimed to be AF in all their essential couplings, which is to be welcomed. If there are classically scale-invariant models of this type that undergo perturbative DT, they will have effective field theories below the DT scale that look like Einstein gravity, at least 
so long as they have a positive gravitational constant (non-minimal couplings $\xi$ greater than zero with our conventions.) If such "vacua" were within the basin of attraction of a UV fixed point for an AF theory, then this would be a candidate model for a unified theory of all interactions, including gravity, in which the mass scales would be determined solely by DT. These are obviously very attractive candidates for further exploration. Whether any such model is consistent with unitarity remains an unresolved issue, which can be addressed after finding a model that is acceptable in other respects.

\section{Acknowledgments}

One of us (MBE) would like to thank A. Vainshtein and E. Rabinovici for discussions and to acknowledge the use of xAct [54] and xTras [55], and to thank J. M. Martín-García and his team, as well as R. McNees, for discussions and advice. DRTJ thanks I. Jack for conversations, I. Avramidi for correspondence, KITP (Santa Barbara) for hospitality and financial support, and the Aspen Center for Physics, where part of this work was done, for hospitality. This research was supported in part by the National Science Foundation under Grant No. NSF PHY11-25915 and Grant No. PHYS-1066293.

Note added. After this paper was completed, our attention was drawn to ref. [53] wherein DT in $R^{2}$ gravity with matter is also proposed in the context of the Standard Model. Their treatment differs considerably from ours. We do not believe their results are applicable to de Sitter space, in particular, with regard to their neglect of the GaussBonnett term and its associated $\beta$-function. As they point out, some of their other $\beta$ functions disagree with those in the literature, which we used herein. We thank A. Salvio and A. Strumia for correspondence.

\section{A Gauss-Bonnet relation}

The local Gauss-Bonnet relation is that a linear combination of three quadratic invariants, $C_{\kappa \lambda \mu \nu}^{2}, R_{\mu \nu}^{2}$ and $R^{2}$ is, in four-dimensions, a total derivative. It can be written in a variety of ways [41]:

$$
\begin{aligned}
R^{*} R^{*} & =R_{\kappa \lambda \mu \nu}^{2}-4 R_{\mu \nu}^{2}+R^{2}=C_{\kappa \lambda \mu \nu}^{2}-2 \widehat{R}_{\mu \nu}^{2}+\frac{1}{6} R^{2} \equiv G \\
\widehat{R}_{\mu \nu} & \equiv R_{\mu \nu}-\frac{g_{\mu \nu}}{4} R, \\
R^{* \kappa \lambda \mu \nu} & \equiv \frac{1}{2} \epsilon^{\kappa \lambda \alpha \beta} R^{\mu \nu}{ }_{\alpha \beta}, \quad R^{*} R^{*}=\frac{1}{4} \epsilon^{\kappa \lambda \alpha \beta} \epsilon_{\mu \nu \gamma \delta} R^{\mu \nu}{ }_{\alpha \beta} R^{\gamma \delta}{ }_{\kappa \lambda}, \\
R^{*} R^{*} & =\nabla_{\mu} B^{\mu}, \\
B^{\mu} & \equiv \epsilon^{\mu \nu \gamma \delta} \epsilon_{\rho \sigma}{ }^{\kappa \lambda} \Gamma_{\kappa \nu}^{\rho}\left[\frac{1}{2} R^{\sigma}{ }_{\lambda \gamma \delta}+\frac{1}{3} \Gamma_{\tau \gamma}^{\sigma} \Gamma_{\lambda \delta}^{\tau}\right] .
\end{aligned}
$$

The current $B^{\mu}$ is not really a vector under diffeomorphisms; it transforms like a connection, but locally, this is irrelevant. In the literature, sometimes the combination $W \equiv R_{\mu \nu}^{2}-$ $R^{2} / 3=\widehat{R}_{\mu \nu}^{2}-R^{2} / 12$ appears, so that $G=C_{\kappa \lambda \mu \nu}^{2}-2 W$.

The global Gauss-Bonnet formula relates the integral of $R^{*} R^{*}$ to the Euler characteristic $\chi$,

$$
\int_{M} d^{4} x \sqrt{g} R^{*} R^{*}+\int_{\partial M} d^{3} x \sqrt{\gamma} B^{\mu} n_{\mu}=32 \pi^{2} \chi
$$


where it has been assumed that $M$ is an orientable, differentiable manifold in fourdimensions, and $\partial M$ represents its possible boundaries. $\left(\gamma_{\kappa \lambda}\right.$ is the push-forward metric on the surface induced by $g_{\mu \nu}$, and $n^{\mu}$ is the outward pointing normal.) The Euler number $\chi=2-2 g$, where $g$ is the genus (number of "handles".) The genus of the sphere $S^{4}$ is zero, so it has $\chi=2$. This relation is very general and, with appropriate modifications of the left-hand side, can be generalized to manifolds and non-smooth surfaces. (It can even be defined topologically without reference to a metric.)

\section{B Background field method}

In this appendix, we review the background field method very briefly, ${ }^{25}$ since we need to refer to a few results in the text. We shall employ DeWitt's condensed notation [59], using a single index to denote all indices, including spacetime $x^{\mu}$ or other continuous parameters. Repeated indices are (usually) summed or integrated over.

The effective action $\Gamma\left[\phi_{i}\right]=S\left[\phi_{i}\right]+\Delta \Gamma\left[\phi_{i}\right]$ includes all quantum corrections $\Delta \Gamma\left[\phi_{i}\right]$ to the classical action $S\left[\phi_{i}\right] . \Delta \Gamma\left[\phi_{i}\right]$ may be defined formally in terms of an integrodifferential equation as follows, in a straightforward generalization of the original path integral treatment of the effective potential [60]. In the classical action, the fields of the theory are shifted $\phi_{i} \rightarrow \phi_{i}+h_{i}$, and the resulting change in the classical action beyond first order in $h_{i}$ is calculated:

$$
\Delta S\left[\phi_{i}, h_{i}\right]=S\left[\phi_{i}+h_{i}\right]-S\left[\phi_{i}\right]-h_{j} \frac{\delta S\left[\phi_{i}\right]}{\delta \phi_{j}} .
$$

Then one defines an auxiliary functional $\Delta \mathcal{G}\left[\phi_{i} ; K_{i}\right]$ by

$$
e^{-\Delta \mathcal{G}\left[\phi_{i} ; K_{i}\right]}=\int_{\mathcal{B}} \mathcal{D} h_{i} e^{-\Delta S\left[\phi_{i}, h_{i}\right]-h_{k} K^{k}},
$$

where $K^{i}$ is initially an arbitrary "source function." Then it can be shown that

$$
\frac{\delta \Delta \mathcal{G}\left[\phi_{i} ; K_{i}\right]}{\delta K_{j}}=0, \text { when } K^{j}\left[\phi_{i}\right]=-\frac{\delta \Delta \Gamma\left[\phi_{i}\right]}{\delta \phi_{j}}, \text { and }
$$

$$
\text { for that value of } K^{j}\left[\phi_{i}\right], \Delta \mathcal{G}\left[\phi_{i} ; K_{j}\left[\phi_{i}\right]\right]=\Delta \Gamma\left[\phi_{i}\right] \text {. }
$$

The interpretation of the expression eq. (B.2) is that $\Delta \mathcal{G}\left[\phi_{i} ; K_{i}\right]$ is the generating functional of 1PI Green's functions in $h_{i}$ for a given background field $\phi_{i}$. These can in principle be evaluated. Then the function $\Delta \mathcal{G}\left[\phi_{i} ; K_{i}\right]$ can be used to choose a source function for a given $\phi_{i}$ so that the one-point function for $h_{i}$ vanishes. (Thus, the background $\phi_{i}$ is selfconsistent.) For that source function $K_{j}\left[\phi_{i}\right]$, then $\Delta \mathcal{G}\left[\phi_{i} ; K_{j}\left[\phi_{i}\right]\right]=\Delta \Gamma\left[\phi_{i}\right]$, the quantum corrections to the classical action.

At first glance, this argument seems circular, but it is in fact well-suited to calculations in perturbation theory. By construction, when expanded in powers of $h_{j}$, the lowest order contribution to $\Delta S\left[\phi_{i}, h_{i}\right]$ is quadratic in $h_{i}$. This determines the propagator for $h_{i}$ as a

\footnotetext{
${ }^{25}$ Early reviews are presented in refs. [56-58] and more recent summaries in refs. [5, 6].
} 
function of the background field. To this order, it gives the well-known correction to the effective action equal to

$$
\Gamma^{(1)}\left[\phi_{i}\right]=\frac{1}{2} \log \operatorname{Det}\left[\frac{\delta^{2} S}{\delta \phi_{i} \delta \phi_{j}}\right]=\frac{1}{2} \operatorname{Tr} \log \left[\frac{\delta^{2} S}{\delta \phi_{i} \delta \phi_{j}}\right] .
$$

There are numerous technical obstacles to implementing this machinery, all of which have been overcome or circumvented. The QFT defined by eqs. (B.1), (B.3) generally requires renormalization, so a cutoff must be introduced. If the theory is renormalizable in the traditional sense, then, in the simplest cases, the action $S$ will contain a finite number of independent monomials in the fields along with their associated coupling constants. In more complicated cases, such as gravity, it may contain an infinite number of terms whose relation to each other is prescribed by a symmetry, i.e., the number of coupling constants does not increase. If it is an effective field theory, then $S$ will contain as many terms (and coupling constants) as are necessary in order to achieve a given degree of accuracy. Regardless, the fluctuations contributing to eq. (B.5) may include negative or zero eigenvalues. Negative modes suggest either that the theory is ill-defined (such as the flat-space $\phi^{3}$-model) or that the background chosen is not self-consistent and must be modified. Zero modes are "flat directions" in the space of fields $h_{i}$, which may be the result of a symmetry or may be resolved by higher order terms in the expansion in $h_{i}$. In any case, it must be determined whether or not $\Delta S\left[\phi_{i}, h_{i}\right]$ is bounded from below as a function of $h_{i}$ or not. In order to interpret the "classical" action in terms of renormalized fields and couplings rather than "bare" quantities, the fields and coupling constants are rescaled in such a way as to render the quantum corrections to Green's functions finite. This is usually expressed by saying that the action includes local counterterms chosen as functions of the cutoff as needed. This makes the determination of stability even more difficult and provisional because it is insufficient to determine simply that $\Delta S\left[\phi_{i}, h_{i}\right] \geq 0$ for bare fields and couplings but must be true for the renormalized fields and couplings, which depend on the renormalisation scale. Ultimately, it is the finite effective action including quantum corrections that needs to be analyzed to determine stability and, in some cases, such as the ones considered in this paper, at certain scales the size of the quantum corrections can be as large as the "classical" corrections. As mentioned in the text, one may even encounter instabilities at one scale that do not persist at other scales. Finally, since the effective action is nonlocal, the criteria for the existence of a sensible background ("vacuum") and stability is not so easily established generically.

In gauge theories, one must introduce gauge-fixing terms in order to obtain sensible Feynman rules, so the effective action is gauge dependent except on-shell where $\delta \Gamma / \delta \phi_{i}=0$. If the gauge-fixing terms are cleverly chosen, one can maintain gauge invariance of the effective action, but that does not mean that they are independent of all gauge-fixing parameters. The ambiguous choice of effective action can make the determination of the stability of a QFT off-shell in principle problematic. At one-loop order, most definitions of a "self-consistent" background field do agree, so AF models may not suffer from such ambiguities concerning their UV behavior. 


\section{Global scale invariance}

In this appendix, we shall review how scale invariance in eq. (5.1) comes about. The scaling symmetry is

$$
x^{\mu} \rightarrow \widehat{x^{\mu}}=e^{-\alpha} x^{\mu}, \quad \phi(x) \rightarrow \widehat{\phi}(\widehat{x})=e^{\alpha} \phi(x), \quad g_{\mu \nu}(x) \rightarrow \widehat{g_{\mu \nu}}(\widehat{x})=g_{\mu \nu}(x),
$$

for arbitrary real $\alpha$. Unlike with general coordinate transformations, the invariant length is rescaled,

$$
d s^{2}=g_{\mu \nu}(x) d x^{\mu} d x^{\nu} \rightarrow d \widehat{s}^{2}=\widehat{g_{\mu \nu}}(\widehat{x}) \widehat{d x^{\mu}} \widehat{d x^{\nu}}=\exp (-2 \alpha) d s^{2} .
$$

In contrast, diffeomorphism invariance corresponds to metric transformations leaving scalars invariant and covariant lengths unchanged:

$$
x \rightarrow x^{\prime}(x), \phi(x) \rightarrow \phi^{\prime}\left(x^{\prime}\right)=\phi(x), d s^{2}=g_{\mu \nu}(x) d x^{\mu} d x^{\nu}=g_{\mu \nu}{ }^{\prime}\left(x^{\prime}\right) d x^{\mu \prime} d x^{\nu \prime}=d s^{\prime 2},
$$

where

$$
g_{\mu \nu}{ }^{\prime}\left(x^{\prime}\right)=\frac{\partial x^{\lambda}}{\partial x^{\prime \mu}} \frac{\partial x^{\sigma}}{\partial x^{\prime \nu}} g_{\lambda \sigma}(x) .
$$

Thus if we make the scale transformation corresponding to eq. (C.1), followed by the general coordinate transformation corresponding to

$$
\widehat{x^{\mu}} \rightarrow x^{\prime \mu}=e^{\alpha} \widehat{x^{\mu}}
$$

it is easy to see that we generate a transformation precisely of the form eq. (5.1), with $x^{\prime}=x$. Thus for a theory which is both scale invariant and general coordinate invariant, we can use this (more convenient) form.

\section{Stability of one-loop effective action}

In order to calculate the one-loop effective action using the background field method of appendix B, we must first form $\Delta S$, eq. (B.1). We write the metric as $g_{\mu \nu} \equiv g_{\mu \nu}^{B}+h_{\mu \nu}$ and the scalar field as $\phi=\phi_{0}+\delta \phi$, where $g_{\mu \nu}^{B}$ is the de Sitter background metric in a convenient choice of coordinates associated with a constant curvature $R_{0}$, and $\phi_{0}$ is the background value of the scalar field. In tree approximation, even though $R_{0}$ and $\phi_{0}$ are undetermined, their ratio is fixed to be $r=\phi_{0}^{2} / R_{0}=\xi(\mu) / \lambda(\mu)$. We shall assume this to be the case, i.e., we restrict our attention to the fluctuations on-shell in order to avoid discussing the complications associated with gauge-fixing. The fluctuations $h_{\mu \nu}$ are decomposed as $[3,5]$

$$
h_{\mu \nu}=h_{\mu \nu}^{\perp}+\nabla_{\{\mu} \mathcal{E}_{\nu\}}+\frac{g_{\mu \nu}^{B}}{4} \varphi
$$

where $h_{\mu \nu}^{\perp}$ is the spin-two projection of $h_{\mu \nu}$ (traceless and transverse, $\nabla^{\mu} h_{\mu \nu}^{\perp}=0$, ) $\mathcal{E}_{\mu}$ is a four-vector, and $\varphi$ is a scalar. Indices are raised and lowered using the background metric $g_{\mu \nu}^{B}$, and the implied connection is with respect to the background metric. If we decompose $\mathcal{E}_{\mu}$ into its transverse (spin one) and longitudinal parts, $\mathcal{E}_{\mu} \equiv \mathcal{E}_{\mu}^{\perp}+\nabla_{\mu} \sigma / 2$, and define $h \equiv$ $g^{B \mu \nu} h_{\mu \nu}$, then we find that $\varphi=h-\square \sigma$. Under a gauge transformation, $\delta h_{\mu \nu}=\nabla_{\{\mu} \Theta_{\nu\}}$, 
then $h_{\mu \nu}^{\perp}$ and $\varphi$ are gauge invariant, and, decomposing $\Theta_{\mu} \equiv \Theta_{\mu}^{\perp}+\nabla_{\mu} \Theta, \delta \mathcal{E}_{\mu}^{\perp}=\Theta_{\mu}^{\perp}$, and $\delta \sigma=2 \Theta, \delta h=2 \square \Theta$. We shall work "on-shell" so that the gauge-dependent modes will not enter. For that matter, we could choose the "unitary gauge" where $\sigma=0, \mathcal{E}_{\mu}^{\perp}=0$.

With this notation, then we find to second order in the fluctuations,

$$
\begin{aligned}
\Delta S^{(2)}= & \int d^{4} x \sqrt{g^{B}}\left[\frac{1}{2}\left(\delta \phi \Delta_{0}\left(2 \xi R_{0}\right) \delta \phi\right)-\delta \phi \frac{3 \xi \phi_{0}}{4} \Delta_{0}\left(-\frac{R_{0}}{3}\right) \varphi+\delta^{(2)} \mathcal{L}_{\mathrm{ho}}\right], \\
\delta^{(2)} \mathcal{L}_{\mathrm{ho}}= & {\left[\frac{3}{16 b} \varphi \Delta_{0}\left(-\frac{b \xi \phi_{0}^{2}}{4}\right) \Delta_{0}\left(-\frac{R_{0}}{3}\right) \varphi\right.} \\
& \left.\quad+\frac{1}{4 a} \bar{h}_{\mu \nu}^{\perp} \Delta_{2}\left(\frac{a \xi \phi_{0}^{2}}{2}+\frac{R_{0}}{3}(1-2 w)\right) \Delta_{2}\left(\frac{R_{0}}{6}\right) \bar{h}^{\perp \mu \nu}\right]
\end{aligned}
$$

where $\Delta_{j}(X) \equiv-\square_{j}+X$ acting on the constrained field of spin $j$. Expanding in eigenfunctions of the Laplacian on the sphere, we have eigenvalues [3]

$$
\square_{j} \equiv \rho_{0}^{2} \bar{\lambda}_{n}^{(j)}, \quad \bar{\lambda}_{n}^{(j)} \equiv n(n+3)-j, \quad d_{n}^{(j)} \equiv \frac{2 j+1}{6}(2 n+3)[n(n+3)-j(j+1)+2],
$$

where $\rho_{0}^{2} \equiv R_{0} / 12, \bar{\lambda}_{n}^{(j)}$ is the eigenvalue on the unit sphere $S^{4}$, and $d_{n}^{(j)}$ is the degree of degeneracy of the eigenvalue $\bar{\lambda}_{n}^{(j)}$. Then, after integration over $S^{4}$, we find for the tensor modes ${ }^{26}$

$$
\Delta S^{(2)}=\frac{1}{4 a} \sum_{n=2}^{\infty} d_{n}^{(2)}\left[\frac{6 \xi^{2}}{y}+4(1-2 w)+\bar{\lambda}_{n}^{(2)}\right]\left[2+\bar{\lambda}_{n}^{(2)}\right]\left(\bar{h}_{n}^{\perp}\right)^{2} .
$$

For convergence of integration over the large $n$-modes, we must have $a>0$. There will be neither negative nor zero modes provided the $n=2$ mode, $\bar{h}_{2}^{\perp}$, has positive coefficient. This requires $w<3 / 2+3 \xi^{2} /(4 y)$. We required $y>0$ for stability of the minimum at $r=r_{0}$. The couplings are to be evaluated at some convenient scale $\mu$ where these inequalities are satisfied.

Returning to eq. (D.2a), we find for the conformal scalar modes,

$$
\begin{aligned}
& \Delta S^{(2)}=\frac{12 \xi}{a y} \sum_{n=0}^{\infty} d_{n}^{(0)}\left[\frac{1}{2}(24 \xi\right.\left.+\bar{\lambda}_{n}^{(0)}\right)\left(\frac{\delta \phi_{n}}{\phi_{0}}\right)^{2}-\frac{3 \xi}{4}\left(-4+\bar{\lambda}_{n}^{(0)}\right)\left(\frac{\delta \phi_{n}}{\phi_{0}}\right) \varphi_{n} \\
&\left.+\left(\frac{w y}{64 \xi}\right)\left(-\frac{3 \xi^{2}}{w y}+\bar{\lambda}_{n}^{(0)}\right)\left(-4+\bar{\lambda}_{n}^{(0)}\right) \varphi_{n}^{2}\right]
\end{aligned}
$$

In order that the large- $n$ modes be positive, it is necessary that $w>0$ and $\xi>0$.

The $n=0$ mode has coefficient $d_{0}^{(0)}=1$ times

$$
\frac{36 \xi^{2}}{a y}\left[4\left(\frac{\delta \phi_{0}}{\phi_{0}}\right)^{2}+\left(\frac{\delta \phi_{0}}{\phi_{0}}\right) \varphi_{0}+\frac{1}{16} \varphi_{0}^{2}\right]
$$

This mixing matrix has one positive eigenvalue $\left(585 \xi^{2} / \lambda\right)$ and one zero eigenvalue, a flat direction. The zero mode is easily understood. Classically, scale invariance is broken

\footnotetext{
${ }^{26}$ This is the same as the results in $[3,5]$ with the appropriate assignment to their masses $m_{0}^{2}, m_{2}^{2}$.
} 
by the background field, and, since this calculation of fluctuations represents simply an expansion of the classical action about a fixed background, there must be a Goldstone boson associated with spontaneous breaking of scale invariance. Removing the zero mode, we get a contribution from the positive eigenvalue to the one-loop correction to the effective action. The QFT explicitly breaks scale invariance owing to the running of the couplings, so we can hope that this zero mode is lifted in higher order, and indeed, at two loops, it obtains a contribution from $C^{(2)} \neq 0$. This classical zero mode thus gets a mass as a result of the anomalous scale invariance. Requiring its mass $^{2}$ to be positive gives a minimum of the action and removes the flat direction.

The next eigenvalue $(n=1)$ has $\bar{\lambda}_{1}=4$ and $d_{1}^{(0)}=5$. The fluctuations are then

$$
\frac{12 \xi}{a y}\left[2(6 \xi+1)\left(\frac{\delta \phi_{1}}{\phi_{0}}\right)^{2}\right] \text {. }
$$

As in the pure gravity case, the coefficient of $\varphi_{1}^{2}$ vanishes, as well as the cross term $\varphi_{1} \delta \phi_{1}$. Thus, we continue to find five zero modes associated with the vanishing of contributions from the $\varphi_{1}$ conformal mode. The other eigenvalue is positive, $24 \xi(6 \xi+1) /(a y)>0$ for $\xi>0$, which we require anyway in order that the gravitational constant $\xi \phi_{0}^{2}$ be positive. We do not understand the reason why these five zero modes persist, i.e., we do not understand this flat direction as the result of a symmetry, broken or unbroken, and we have the feeling that we may be missing something. Unlike the dilaton mode, we do not know whether it remains flat in higher order or just what happens.

In sum, the constraints on the couplings in order that all modes be nonnegative are that all four couplings $a, w, \xi, y$ be positive at some scale and that $w<3 / 2+3 \xi^{2} /(4 y)$.

\section{E One-loop beta-functions}

We have taken results for $\beta_{a, b, \varepsilon}^{\text {ho }}$ from ref. [4], which corrects earlier results of Fradkin and Tseytlin $([2,3])$ for $\beta_{b}$. At one-loop order, the effect of matter on the gravitational betafunctions is simply to add another term, so that $\beta_{a}, \beta_{b}$ (or $\beta_{w}$,) and $\beta_{\varepsilon}$ become sums $\beta_{i}=\beta_{i}^{\text {ho }}+\beta_{i}^{\text {mat }}$. These results also follow from gravitational trace anomaly calculations and are well known: see for example ref. [61]. For the gravitational contributions to the matter $\beta$-functions $\beta_{\lambda, \xi}$, we have used ref. [6] and references therein. We summarise the $\beta$-functions below in our notation:

$$
\begin{array}{rlrl}
\frac{1}{\kappa} \beta_{\varepsilon}^{\text {ho }} & =-\frac{196}{45}, & \frac{1}{\kappa} \beta_{\varepsilon}^{\text {mat }} & =-\frac{1}{360}\left[N_{0}+11 N_{1 / 2}+62 N_{1}^{0}+63 N_{1}\right] \\
\frac{1}{\kappa} \beta_{a}^{\text {ho }} & =-\frac{133}{10} a^{2}, & \frac{1}{\kappa} \beta_{a}^{\text {mat }}=-\frac{a^{2}}{60}\left[N_{0}+6 N_{1 / 2}+12 N_{1}^{0}+13 N_{1}\right] \\
\frac{1}{\kappa} \beta_{b}^{\text {ho }}=-\frac{5}{3}\left[2 a^{2}-3 a b+\frac{b^{2}}{4}\right], & \frac{1}{\kappa} \beta_{b}^{\text {mat }}=-\frac{b^{2}}{24}\left[(1+6 \xi)^{2} N_{0}+N_{1}\right] ; \\
\frac{1}{\kappa} \beta_{w}^{\text {ho }}=\frac{10 a}{3}\left[w^{2}-\frac{549}{100} w+\frac{1}{8}\right], & \\
\frac{1}{\kappa} \beta_{w}^{\text {mat }}=-\frac{a}{60} w\left[N_{0}+6 N_{1 / 2}+12 N_{1}^{0}+13 N_{1}\right]+\frac{a}{24}\left[(1+6 \xi)^{2} N_{0}+N_{1}\right]
\end{array}
$$


where $N_{0}$ denotes the number of (real) scalars; $N_{1 / 2}$, DIRAC fermions; ${ }^{27} N_{1}^{0}$, massless vectors; $N_{1}$, massive vectors. (For chiral or Majorana fermions, the coefficients of $N_{1 / 2}$ would be half those given above). The parameter $\xi$ represents the non-minimal coupling of a real scalar; in general, one may have a sum of such couplings. For a general theory we may write (at one loop) $\beta_{a}=-\kappa \beta_{2} a^{2}$, and $\beta_{\varepsilon}=-\kappa \beta_{1}$, with positive constants $\beta_{2}, \beta_{1}$. It can be shown [22] that the leading contribution to $\varepsilon$ is determined to be $\varepsilon=\varepsilon_{0}-\beta_{1} /\left(\beta_{2} a\right)$ where $\varepsilon_{0}$ is a scale-independent constant.

The one-loop beta-functions for the matter couplings obviously depend on the particular model. For the single, real scalar action eq. (1.2) with couplings $\xi$ and $\lambda$, they are

$$
\begin{array}{ll}
\frac{1}{\kappa} \beta_{\xi}^{\mathrm{ho}}=-a \xi\left[\frac{3 \xi^{2}}{2}+4 \xi-3+\frac{10 w}{3}-\frac{1}{w}\left(\frac{9 \xi^{2}}{4}+5 \xi-1\right)\right], & \frac{1}{\kappa} \beta_{\xi}^{\mathrm{mat}}=(6 \xi+1) \lambda \\
\frac{1}{\kappa} \beta_{\lambda}^{\mathrm{ho}}=\frac{a^{2} \xi^{2}}{2}\left[5+\frac{(6 \xi+1)^{2}}{4 w^{2}}\right]-a \lambda\left[5+3 \xi^{2}-\left(\frac{1+12 \xi+33 \xi^{2}}{2 w}\right)\right], & \frac{1}{\kappa} \beta_{\lambda}^{\mathrm{mat}}=18 \lambda^{2} .
\end{array}
$$

This system of equations for $a, w, \xi, \lambda$ are rather complicated, but they can be somewhat simplified by introducing the variable $u \equiv\left(1 / \beta_{2}\right) \log \left(a_{0} / a(\mu)\right)$. Then these equations may be written as in eq. (5.22) in the text.

Open Access. This article is distributed under the terms of the Creative Commons Attribution License (CC-BY 4.0), which permits any use, distribution and reproduction in any medium, provided the original author(s) and source are credited.

\section{References}

[1] K.S. Stelle, Renormalization of higher derivative quantum gravity, Phys. Rev. D 16 (1977) 953 [INSPIRE].

[2] E.S. Fradkin and A.A. Tseytlin, Renormalizable asymptotically free quantum theory of gravity, Phys. Lett. B 104 (1981) 377 [INSPIRE].

[3] E.S. Fradkin and A.A. Tseytlin, Renormalizable asymptotically free quantum theory of gravity, Nucl. Phys. B 201 (1982) 469 [INSPIRE].

[4] I.G. Avramidi and A.O. Barvinsky, Asymptotic freedom in higher derivative quantum gravity, Phys. Lett. B 159 (1985) 269 [INSPIRE].

[5] I.G. Avramidi, Heat kernel and quantum gravity, Lecture Notes in Physics volume 64, Springer, Germany (2000.

[6] I.L. Buchbinder, S.D. Odintsov and I.L. Shapiro, Effective action in quantum gravity, IOP, Bristol U.K. (1992).

[7] W.A. Bardeen, On naturalness in the standard model, FERMILAB-CONF-95-391 (1995).

[8] W.A. Bardeen, private communication.

[9] R. Foot, A. Kobakhidze, K.L. McDonald and R.R. Volkas, A solution to the hierarchy problem from an almost decoupled hidden sector within a classically scale invariant theory, Phys. Rev. D 77 (2008) 035006 [arXiv:0709.2750] [INSPIRE].

\footnotetext{
${ }^{27}$ Not, as stated in ref. [5], two-component ones.
} 
[10] R. Foot, A. Kobakhidze and R.R. Volkas, Stable mass hierarchies and dark matter from hidden sectors in the scale-invariant standard model, Phys. Rev. D 82 (2010) 035005 [arXiv: 1006.0131] [INSPIRE].

[11] W. Altmannshofer, W.A. Bardeen, M. Bauer, M. Carena and J.D. Lykken, Light dark matter, naturalness and the radiative origin of the electroweak scale, JHEP 01 (2015) 032 [arXiv: 1408.3429] [INSPIRE].

[12] G. 't Hooft, Naturalness, chiral symmetry, and spontaneous chiral symmetry breaking, NATO Adv. Study Inst. Ser. B Phys. 59 (1980) 135 [inSPIRE].

[13] S.R. Coleman and E.J. Weinberg, Radiative corrections as the origin of spontaneous symmetry breaking, Phys. Rev. D 7 (1973) 1888 [inSPIRE].

[14] S. Carlip, Quantum gravity: a progress report, Rept. Prog. Phys. 64 (2001) 885 [gr-qc/0108040] [INSPIRE].

[15] N.H. Barth and S.M. Christensen, Quantizing fourth order gravity theories. 1. The functional integral, Phys. Rev. D 28 (1983) 1876 [InSPIRE].

[16] G.W. Gibbons and S.W. Hawking, Action integrals and partition functions in quantum gravity, Phys. Rev. D 15 (1977) 2752 [inSPIRE].

[17] N.H. Barth, The fourth order gravitational action for manifolds with boundaries, Class. Quant. Grav. 2 (1985) 497 [INSPIRE].

[18] E.T. Tomboulis, Exact relation between Einstein and quadratic quantum gravity, Phys. Lett. B 389 (1996) 225 [hep-th/9601082] [INSPIRE].

[19] S.D. Odintsov, The parametrization invariant and gauge invariant effective actions in quantum field theory, Fortsch. Phys. 38 (1990) 371 [INSPIRE].

[20] D.M. Capper and D. Kimber, An ambiguity in one loop quantum gravity, J. Phys. A 13 (1980) 3671 [inSPIRE].

[21] S.A. Brunini and M. Gomes, The Gauss-Bonnet identity in fourth order gravity, Mod. Phys. Lett. A 8 (1993) 1977 [INSPIRE].

[22] collaboration, M.B. Einhorn and D.R.T. Jones, The Gauss-Bonnet coupling constant in classically scale-invariant gravity, arXiv:1412.5572 [INSPIRE].

[23] I. Jack and H. Osborn, Background field calculations in curved space-time. 1. General formalism and application to scalar fields, Nucl. Phys. B 234 (1984) 331 [INSPIRE].

[24] I. Jack, Background field calculations in curved space-time. 3. Application to a general gauge theory coupled to fermions and scalars, Nucl. Phys. B 253 (1985) 323 [INSPIRE].

[25] J.L. Cardy, Is there a c theorem in four-dimensions?, Phys. Lett. B 215 (1988) 749 [INSPIRE].

[26] S.J. Hathrell, Trace anomalies and $\lambda \phi^{4}$ theory in curved space, Annals Phys. 139 (1982) 136 [INSPIRE].

[27] D.Z. Freedman and H. Osborn, Constructing a c function for SUSY gauge theories, Phys. Lett. B 432 (1998) 353 [hep-th/9804101] [INSPIRE].

[28] I. Jack and H. Osborn, Analogs for the $c$ theorem for four-dimensional renormalizable field theories, Nucl. Phys. B 343 (1990) 647 [INSPIRE]. 
[29] Z. Komargodski and A. Schwimmer, On renormalization group flows in four dimensions, JHEP 12 (2011) 099 [arXiv:1107.3987] [INSPIRE].

[30] Z. Komargodski, The constraints of conformal symmetry on RG flows, JHEP 07 (2012) 069 [arXiv:1112.4538] [INSPIRE].

[31] I. Jack and H. Osborn, Constraints on $R G$ flow for four dimensional quantum field theories, Nucl. Phys. B 883 (2014) 425 [arXiv: 1312.0428] [inSPIRE].

[32] I. Jack and C. Poole, The a-function for gauge theories, JHEP 01 (2015) 138 [arXiv: 1411.1301] [INSPIRE].

[33] A.O. Barvinsky, The a-theorem and temperature of the CMB temperature in cosmology, arXiv: 1305.4223 [INSPIRE].

[34] G. 't Hooft, Dimensional regularization and the renormalization group, Nucl. Phys. B 61 (1973) 455 [inSPIRE].

[35] M. Rinaldi, G. Cognola, L. Vanzo and S. Zerbini, Reconstructing the inflationary $f(R)$ from observations, JCAP 08 (2014) 015 [arXiv:1406.1096] [INSPIRE].

[36] M. Rinaldi, G. Cognola, L. Vanzo and S. Zerbini, Inflation in scale-invariant theories of gravity, arXiv:1410.0631 [INSPIRE].

[37] C. Kounnas, D. Lüst and N. Toumbas, $R^{2}$ inflation from scale invariant supergravity and anomaly free superstrings with fluxes, Fortsch. Phys. 63 (2015) 12 [arXiv:1409.7076] [INSPIRE].

[38] E. Elizalde, S.D. Odintsov and A. Romeo, Improved effective potential in curved space-time and quantum matter, higher derivative gravity theory, Phys. Rev. D 51 (1995) 1680 [hep-th/9410113] [INSPIRE].

[39] E. Elizalde et al., GUTs in curved space-time: running gravitational constants, Newtonian potential and the quantum corrected gravitational equations, Phys. Rev. D 52 (1995) 2202 [hep-th/9504014] [INSPIRE].

[40] E.S. Fradkin and A.A. Tseytlin, One loop effective potential in gauged $O(4)$ supergravity, Nucl. Phys. B 234 (1984) 472 [INSPIRE].

[41] E.S. Fradkin and A.A. Tseytlin, Conformal supergravity, Phys. Rept. 119 (1985) 233 [INSPIRE].

[42] A.Y. Kamenshchik and C.F. Steinwachs, Frame dependence of quantum corrections in cosmology, arXiv:1408.5769 [inSPIRE].

[43] D. Buttazzo et al., Investigating the near-criticality of the Higgs boson, JHEP 12 (2013) 089 [arXiv: 1307.3536] [INSPIRE].

[44] A. Andreassen, W. Frost and M.D. Schwartz, Consistent use of the standard model effective potential, Phys. Rev. Lett. 113 (2014) 241801 [arXiv:1408.0292] [INSPIRE].

[45] L. Di Luzio and L. Mihaila, On the gauge dependence of the standard model vacuum instability scale, JHEP 06 (2014) 079 [arXiv:1404.7450] [INSPIRE].

[46] F.L. Bezrukov and M. Shaposhnikov, The standard model Higgs boson as the inflaton, Phys. Lett. B 659 (2008) 703 [arXiv:0710.3755] [INSPIRE].

[47] A.O. Barvinsky, A.Y. Kamenshchik and A.A. Starobinsky, Inflation scenario via the standard model Higgs boson and LHC, JCAP 11 (2008) 021 [arXiv: 0809.2104] [INSPIRE]. 
[48] A. De Simone, M.P. Hertzberg and F. Wilczek, Running inflation in the standard model, Phys. Lett. B 678 (2009) 1 [arXiv:0812.4946] [INSPIRE].

[49] F. Bezrukov and M. Shaposhnikov, Standard model Higgs boson mass from inflation: two loop analysis, JHEP 07 (2009) 089 [arXiv:0904.1537] [INSPIRE].

[50] A.O. Barvinsky, A.Y. Kamenshchik, C. Kiefer, A.A. Starobinsky and C. Steinwachs, Asymptotic freedom in inflationary cosmology with a non-minimally coupled Higgs field, JCAP 12 (2009) 003 [arXiv:0904.1698] [INSPIRE].

[51] E.J. Weinberg and A.Q. Wu, Understanding complex perturbative effective potentials, Phys. Rev. D 36 (1987) 2474 [INSPIRE].

[52] H. Yamagishi, Coupling constant flows and dynamical symmetry breaking, Phys. Rev. D 23 (1981) 1880 [inSPIRE].

[53] A. Salvio and A. Strumia, Agravity, JHEP 06 (2014) 080 [arXiv: 1403.4226] [InSPIRE].

[54] J. M. Martín-García, xAct: efficient tensor computer algebra for Mathematica, http://www.xact.es/.

[55] T. Nutma, xTras: a field-theory inspired xAct package for mathematica, Comput. Phys. Commun. 185 (2014) 1719 [arXiv:1308.3493] [InSPIRE].

[56] L.F. Abbott, Introduction to the background field method, Acta Phys. Polon. B 13 (1982) 33 [INSPIRE].

[57] A.O. Barvinsky and G.A. Vilkovisky, The generalized Schwinger-Dewitt technique in gauge theories and quantum gravity, Phys. Rept. 119 (1985) 1 [INSPIRE].

[58] B.S. DeWitt, The effective action, in Quantum field theory and quantum statistics, I.A. Batalin et al. eds., Hilger U.K. (1987).

[59] B.S. DeWitt, Dynamical theory of groups and fields, Conf. Proc. C 630701 (1964) 585 [Les Houches Lect. Notes 13 (1964) 585].

[60] R. Jackiw, Functional evaluation of the effective potential, Phys. Rev. D 9 (1974) 1686 [INSPIRE].

[61] N.D. Birrell and P.C.W. Davies, Quantum fields in curved space, Cambridge University Press, Cambridge U.K. (1982). 